\title{
Comparison Theorems in Riemannian Geometry
}

\author{
J.-H. Eschenburg
}

\section{Introduction}

The subject of these lecture notes is comparison theory in Riemannian geometry: What can be said about a complete Riemannian manifold when (mainly lower) bounds for the sectional or Ricci curvature are given? Starting from the comparison theory for the Riccati ODE which describes the evolution of the principal curvatures of equidistant hypersurfaces, we discuss the global estimates for volume and length given by Bishop-Gromov and Toponogov. An application is Gromov's estimate of the number of generators of the fundamental group and the Betti numbers when lower curvature bounds are given. Using convexity arguments, we prove the "soul theorem" of Cheeger and Gromoll and the sphere theorem of Berger and Klingenberg for nonnegative curvature. If lower Ricci curvature bounds are given we exploit subharmonicity instead of convexity and show the rigidity theorems of Myers-Cheng and the splitting theorem of Cheeger and Gromoll. The Bishop-Gromov inequality shows polynomial growth of finitely generated subgroups of the fundamental group of a space with nonnegative Ricci curvature (Milnor). We also discuss briefly Bochner's method.

The leading principle of the whole exposition is the use of convexity methods. Five ideas make these methods work: The comparison theory for the Riccati ODE, which probably goes back to L.Green [15] and which was used more systematically by Gromov [20], the triangle inequality for the Riemannian distance, the method of support function by Greene and $\mathrm{Wu}[16],[17],[34]$, the maximum principle of E.Hopf, generalized by E.Calabi [23], [4], and the idea of critical points of the distance function which was first used by Grove and Shiohama [21]. We have tried to present the ideas completely without being too technical.

These notes are based on a course which I gave at the University of Trento in March 1994. It is a pleasure to thank Elisabetta Ossanna and Stefano Bonaccorsi who have worked out and typed part of these lectures. We also thank Evi Samiou and Robert Bock for many valuable corrections.

Augsburg, September 1994

J.-H. Eschenburg 


\section{Covariant derivative and curvature.}

Notation: By $M$ we always denote a smooth manifold of dimension $n$. For $p \in M$, the tangent space at $p$ is denoted by $T_{p} M$, and $T M$ denotes the tangent bundle. If $M^{\prime}$ is another manifold and $f: M \rightarrow M^{\prime}$ a smooth (i.e. $C^{\infty}$ ) map, its differential at some point $p \in M$ is always denoted by $d f_{p}: T_{p} M \rightarrow T_{f(p)} M^{\prime}$. For $v \in T_{p} M$ we write $d f_{p}(v)=d f_{p} . v=\partial_{v} f$. If $c: I \rightarrow M$ is a (smooth) curve, we denote its tangent vector by $c^{\prime}(t)=d c(t) / d t=d c_{t} .1 \in T_{c(t)} M$ (where $1 \in T_{t} I=\mathbb{R}$ ). If $f: M \rightarrow \mathbb{R}$, then $d f_{p} \in\left(T_{p} M\right)^{*}$. If $M$ is a Riemannian manifold, i.e. there exists a scalar product $<,>$ on any tangent space of $M$, this gives an isomorphism between $T_{p} M$ and $\left(T_{p} M\right)^{*}$; the vector $\nabla f(p)$ corresponding to $d f_{p}$ is called the gradient of $f$.

Let $M$ be a Riemannian manifold. We denote by $<,>$ the scalar product on $M$ and we define the norm of a vector by

$$
\|v\|=\sqrt{\langle v, v\rangle}
$$

the length of a curve $c: I \rightarrow M$ by

$$
L(c)=\int_{I}\left\|c^{\prime}(t)\right\| d t
$$

and the distance between $x, y \in M$ by

$$
|x, y|=\inf \{L(c) ; c: x \rightarrow y\} .
$$

where $c: x \rightarrow y$ means that $c:[a, b] \rightarrow M$ with $c(a)=x$ and $c(b)=y$. If $L(c)=|x, y|$ for some $c: x \rightarrow y$, then $c$ is called shortest. The open and closed metric balls are denoted by $B_{r}(p)$ and $D_{r}(p)$, i.e.

$$
B_{r}(p)=\{x \in M ;|x, p|<r\}, \quad D_{r}(p)=\{x \in M ;|x, p| \leq r\} .
$$

Similarly, we define $B_{r}(A)$ for any closed subset $A \subset M$.

We denote by $\mathbf{X}(M)$ the set of vector fields on $M$.

Definition 1.1 The Levi-Civita covariant derivative

$$
\begin{gathered}
D: \mathbf{X}(M) \times \mathbf{X}(M) \rightarrow \mathbf{X}(M) \\
(X, Y) \rightarrow D_{X} Y,
\end{gathered}
$$

is determined by the following properties holding for all functions $f, g \in C^{\infty}(M)$ and for all vector fields $X, X^{\prime}, Y, Y^{\prime} \in \mathbf{X}(M)$ : 
1. $D_{\left(f X+g X^{\prime}\right)} Y=f D_{X} Y+g D_{X^{\prime}} Y$;

2. $D_{X}\left(f Y+g Y^{\prime}\right)=\left(\partial_{X} f\right) Y+f D_{X} Y+\left(\partial_{X} g\right) Y^{\prime}+g D_{X} Y^{\prime}$

3. $D_{X} Y-D_{Y} X=[X, Y]=$ "Lie bracket";

4. $\partial_{Z}\langle X, Y\rangle=\left\langle D_{Z} X, Y\right\rangle+\left\langle X, D_{Z} Y\right\rangle$.

Definition 1.2 The Riemannian curvature tensor $(X, Y, Z) \mapsto R(X, Y) Z$ is defined as follows:

$$
R(X, Y) Z=D_{X} D_{Y} Z-D_{Y} D_{X} Z-D_{[X, Y]} Z
$$

It satisfies certain algebraic identities ("curvature identities"), namely

$$
<R(X, Y) Z, W>=-<R(Y, X) Z, W>=-<R(X, Y) W, Z>=<R(Z, W) X, Y>
$$

and the Bianchi identity

$$
R(X, Y) Z+R(Y, Z) X+R(Z, X) Y=0
$$

(cf. [29]). In particular,

$$
R_{V}:=R(., V) V
$$

is a self adjoint endomorphism of TM for any vector field $V$ on $M$. Several notions of curvature are derived from this tensor:

1. Sectional curvature $K($,$) : For every linearly independent pair of vectors X, Y \in$ $T_{p} M$

$$
K(X, Y)=\frac{<R(X, Y) Y, X>}{\|X\|^{2}\|Y\|^{2}-<X, Y>^{2}} .
$$

$K$ is defined on the space of two dimensional linear subspaces of $T_{p} M$ (depending only on $\operatorname{span}(X, Y))$.

2. Ricci curvature

$$
\operatorname{Ric}(X, Y)=\operatorname{trace}(Z \mapsto R(Z, X) Y) .
$$

By the curvature identities, $\operatorname{Ric}(X, Y)=\operatorname{Ric}(Y, X)$. Ricci curvature in direction $X$ is given by

$$
\operatorname{Ric}(X)=\operatorname{Ric}(X, X)
$$

where $X$ is a unit vector.

3. Scalar curvature

$$
s=\operatorname{trace}(\operatorname{Ric})=\sum \operatorname{Ric}\left(E_{i}, E_{i}\right)
$$


where $\left\{E_{i}\right\}_{i=1}^{n}$ is a local orthonormal basis.

There is a close relationship between $R_{V}=R(., V) V$ and the sectional curvature: Let $\|V\|=1$. For $X$ orthogonal to $V$ we have

$$
<R_{V} X, X>=<R(X, V) V, X>=K(V, X)\|X\|^{2}
$$

Hence the highest (" $\lambda_{+}$") and lowest (" $\lambda_{-} "$ ) eigenvalues of $R_{V}$ give a bound to $K(V, X)$, since

$$
\lambda_{-}\left(R_{V}\right) \leq \frac{<R_{V} X, X>}{<X, X>} \leq \lambda_{+}\left(R_{V}\right) .
$$

Moreover, $\operatorname{trace}\left(R_{V}\right)=\operatorname{Ric}(V, V)$.

Let us come back to the covariant derivative. It is easy to see that for any $p \in M$, $\left(D_{X} Y\right)_{p}$ depends only on $d Y_{p} . X(p)$ where the vector field $Y$ is considered as a smooth map $Y: M \rightarrow T M$. Therefore, the covariant derivative is also defined if the vector fields $X$ and $Y$ are only partially defined. E.g. if $\gamma: I \rightarrow M$ is a smooth regular curve and $Y$ is a vector field along $\gamma$, i.e. a smooth map $Y: I \rightarrow T M$ with

$$
Y(t) \in T_{\gamma(t)} M
$$

for all $t \in I$ (e.g. $\gamma^{\prime}$ is such a vector field), then

$$
Y^{\prime}(t):=\frac{D Y(t)}{d t}:=D_{\gamma^{\prime}(t)} Y
$$

is defined (just extend $\gamma^{\prime}$ and $Y$ arbitrarly outside $\gamma$ ). Similar, if $\gamma: I_{1} \times \ldots \times I_{k} \rightarrow M$ depends on $k$ variables, we have $k$ partial derivatives $\frac{\partial \gamma}{\partial t_{i}}$ and corresponding covariant derivatives $\frac{D}{\partial t_{i}}(i=1, \ldots, k)$ along $\gamma$. (Formally, a vector field along $\gamma$ is a section of the pull-back bundle $\gamma^{*} T M$, and $D$ induces a covariant derivative on this bundle.)

Definition 1.3 A vector field $Y$ along a curve $\gamma: I \rightarrow M$ is called parallel if $Y^{\prime}=0$. A curve $\gamma$ is a called a geodesic in $M$ if $\gamma^{\prime}$ is parallel, i.e. if

$$
\left(\gamma^{\prime}\right)^{\prime}=D_{\gamma^{\prime}} \gamma^{\prime}=0
$$

(1.1) is a $2^{n d}$ order ODE. In fact, if $x=\left(x^{1}, \ldots, x^{n}\right): M \rightarrow \mathbb{R}^{n}$ is a coordinate chart with $E_{i}=\frac{\partial}{\partial x^{i}}$ and if we put

$$
D_{E_{i}} E_{j}=\Gamma_{i j}^{k} E_{k}
$$


(summation convention!), then $\gamma^{\prime}=\left(\gamma^{i}\right)^{\prime} E_{i}$ where $\gamma^{i}:=x^{i} \circ \gamma$, and

$$
\frac{D}{d t} \gamma^{\prime}=\left(\gamma^{i}\right)^{\prime \prime} E_{i}+\left(\gamma^{j}\right)^{\prime} D_{\gamma^{\prime}} E_{j}
$$

with

$$
D_{\gamma^{\prime}} E_{j}=\gamma^{k} D_{E_{k}} E_{j}=\left(\gamma^{k}\right)^{\prime} \Gamma_{k j}^{i} E_{i}
$$

hence (1.1) is equivalent to

$$
\left(\gamma^{i}\right)^{\prime \prime}+\left(\gamma^{j}\right)^{\prime}\left(\gamma^{k}\right)^{\prime} \Gamma_{k j}^{i}=0
$$

To some extent, Riemannian geometry is the theory of this ODE.

Definition 1.4 For any $v \in T M$ let $\gamma_{v}$ denote the unique geodesic with $\gamma^{\prime}(0)=v$. For $s, t \in \mathbb{R}$ with $|s|$ and $|t|$ small we have $\gamma_{s v}(t)=\gamma_{v}(s t)$ by uniqueness for ODE's. Thus for $v \in T M$ with $\|v\|$ small enough,

$$
\exp (v):=\gamma_{v}(1)
$$

is defined and gives a smooth map exp $:(T M)_{0} \rightarrow M$ where $(T M)_{0}$ is a neighborhood of the zero section of $T M$. This is called the exponential map of $M . M$ is called (geodesically) complete if exp is defined on all of $T M$. Fixing $p \in M$, we put $\exp _{p}=$ $\exp \mid T_{p} M$.

Remark 1.5 The map $\exp _{p}$ is a diffeomorphism near the origin (in fact, $d\left(\exp _{p}\right)_{0}$ is the identity on $\left.T_{p} M\right)$, and it maps all the lines through the origin of $T_{p} M$ onto the geodesics through the point $p \in M$. Thus, $\exp _{p}: T_{p} M \rightarrow M$ can serve a a coordinate map near $p$ ("exponential coordinates") which preserves the covariant derivative at p, i.e. covariant differentiation at $p$ is the same as taking the ordinary derivative at $0 \in T_{p} M$ in exponential coordinates. To see this, identify $M$ and $T_{p} M$ near $p$ via $\exp _{p}$ and consider the ordinary derivative $D^{o}=\partial$ on $T_{p} M$. It satisfies the rules (1.),(2.) and (3.) of the Levi-Civita derivative (but not 4. for the Riemannian metric). Hence the difference $\Gamma=D-\partial$ is a tensor field, i.e. $\Gamma_{X} f Y=f \Gamma_{X} Y$ for all functions $f$ (by 2.), further it is symmetric, i.e. $\Gamma_{X} Y=\Gamma_{Y} X$ (by 3.), and it satisfies $\Gamma_{v} v=0$ for all $v \in T_{p} M$, since $D$ and $\partial$ have the same geodesics at $p$. Thus $\left.\Gamma\right|_{p}=0$. 


\section{Jacobi and Riccati equations; equidistant hypersurfaces.}

Equation (1.1) is a nonlinear ODE which in general cannot be solved explicitly. Therefore, we consider its linearization. This is the ODE satisfied by a variation of solutions of (1.1), i.e. of geodesics. So let $\gamma(s, t)=\gamma_{s}(t)$ be a smooth one-parameter family of geodesics $\gamma_{s}$. Put $V=\frac{\partial \gamma}{\partial t} \in \mathbf{X}\left(\gamma_{s}\right)$ and $J=\frac{\partial \gamma}{\partial s}$. Then $J$ is the variation vector field and $V$ the tangent field of the geodesics $\gamma_{s}$, hence $D_{V} V=O$.

Fig. 1.

Then we have

$$
J^{\prime \prime}=\frac{D}{\partial t} \frac{D}{\partial t} J=\frac{D}{\partial t} \frac{D}{\partial t} \frac{\partial \gamma}{\partial s}
$$

We can interchange the order of differentiation, getting

$$
\begin{gathered}
J^{\prime \prime}=\frac{D}{\partial s} \frac{D}{\partial t} \frac{\partial \gamma}{\partial t}+R(V, J) V \\
J^{\prime \prime}+R(J, V) V=0
\end{gathered}
$$

Equation (2.1) is called Jacobi equation.

Definition 2.1 A vector field $J$ along a geodesic $\gamma$ is called a Jacobi field if it satisfies the Jacobi equation.

Remark 2.2 $J$ is a Jacobi field along $\gamma$ if and only if

$$
J(t)=\left.\frac{d}{d s}\right|_{0} \gamma_{s}(t)
$$


for some one-parameter family of geodesics $\gamma_{s}$ with $\gamma_{0}=\gamma$.

Implication " $\Leftarrow "$ was shown above. To prove the opposite implication, we have to construct the family $\gamma_{s}$. Let $\alpha(s)=\exp _{\gamma(0)} s J(0)$. Let $X$ be a vector field along $\alpha$ such that $X(0)=\gamma^{\prime}(0)$ and $X^{\prime}(0)=J^{\prime}(0)$ and put

$$
\gamma_{s}(t)=\exp _{\alpha(s)} t X(s)
$$

If we put

$$
\tilde{J}=\left.\frac{\partial}{\partial s}\right|_{0} \gamma_{s}
$$

then, by "£", $\tilde{J}$ satisfies the Jacobi equation. Since $\tilde{J}(0)=J(0)$ and

$$
\tilde{J}^{\prime}(0)=\left.\frac{D}{\partial t} \frac{\partial}{\partial s} \gamma\right|_{(0,0)}=\left.\frac{D}{\partial s} \frac{\partial}{\partial t} \gamma\right|_{(0,0)}=\left.\frac{D}{\partial s} X(s)\right|_{0}=X^{\prime}(0)=J^{\prime}(0),
$$

we get $J=\tilde{J}$ by uniqueness of the solution.

Next, we want to split this $2^{\text {nd }}$ degree equation in a system of $1^{\text {st }}$ degree equations. To do this, we embed the 1-parameter family of geodesics describing the Jacobi field into an $(n-1)$-parameter family. I.e. we choose a suitable smooth map

$$
\gamma: S \times I \rightarrow M
$$

where $S$ is an $(n-1)$-dimensional manifold, such that $\gamma_{s}(t)=\gamma(s, t)$ is a geodesic for any $s \in S$. If $\gamma$ is a regular map, then $V=d \gamma\left(\frac{\partial}{\partial t}\right)$ can be viewed as a vector field on an open subset of $M$ with $D_{V} V=0$, and the Jacobi fields $J$ arising from variations in $S$ directions commute with $V$, i.e. we have $[J, V]=0$ or

$$
D_{V} J=A \cdot J
$$

where $A=D V$, i.e. $A \cdot X=D_{X} V$. This is the first of our equations: Knowing $A$, we recieve $J$ by solving a $1^{\text {st }}$ order equation.

It remains to derive an equation for $A$. Let us consider first an arbitrary vector field $V$ on $M$ and let $A=D V$ as before. In general, the covariant derivative of a tensor field $A$ is defined by

$$
\left(D_{V} A\right) X=D_{V}(A X)-A \cdot D_{V}(X)
$$


Hence we have

$$
\begin{aligned}
\left(D_{V} A\right) X & =D_{V} D_{X} V-A\left(D_{X} V+[V, X]\right) \\
& =D_{X} D_{V} V+R(V, X) V+D_{[V, X]} V-A^{2} X-A[V, X] \\
& =D_{X} D_{V} V+R_{V}(X)-A^{2} X
\end{aligned}
$$

Therefore

$$
D_{V} A+A^{2}+R_{V}=D\left(D_{V} V\right) .
$$

If we suppose $D_{V} V=0$ (i.e. the integral curves $\gamma_{s}$ are geodesic), then we get an ODE for $A$, the so called Riccati equation

$$
A^{\prime}+A^{2}+R_{V}=0
$$

Thus we have split the Jacobi equation $J^{\prime \prime}=-R_{V} J$ in two equations as follows:

$$
\begin{gathered}
J^{\prime}=A J \\
A^{\prime}+A^{2}+R_{V}=0 .
\end{gathered}
$$

We note that the second equation can be solved independently of the first.

Let us consider now the important special case where $(D V)^{*}=D V$, that is

$$
<D_{X} V, Y>=<X, D_{Y} V>
$$

for all vector fields $X, Y$. Then $V$ is locally a gradient, i.e. locally $V=\nabla f$ for some function $f: M \rightarrow \mathbb{R}$. Consequently, $\langle V, V\rangle$ is constant, since

$$
\partial_{X}<V, V>=2<D_{X} V, V>=2<D_{V} V, X>=0 .
$$

Thus we may assume that $\langle V, V\rangle=1$. Now let us consider the level hypersurfaces

$$
S_{t}=\{x \in M: f(x)=t\} .
$$

Since $V=\nabla f \neq 0$, the $S_{t}$ are regular hypersurfaces and $\left.V\right|_{S_{t}}$ is the unit normal vector field on $S_{t}$. Thus in this case, our $(n-1)$-parameter family of geodesics $\gamma: S \times I \rightarrow M$ is given by

$$
\gamma(s, t)=\exp \left(t-t_{0}\right) V(s)
$$


where $S=S_{t_{0}}$ for some $t_{0} \in I$, and $f(\gamma(s, t))=t$, or in other words, $S_{t}=\phi_{t}(S)$ where $\phi_{t}(s):=\gamma(s, t)$. Such a family of hypersurfaces $S_{t}$ is called equidistant, and the function $f-t_{0}$ is called the signed distance function of the hypersurface $S=S_{t_{0}}$. In fact we have

$$
\left|f(x)-t_{0}\right|=|x, S|:=\inf _{s \in S}|x, s|
$$

for $x$ in a small neighborhood of $S$. Namely, if $c:[a, b] \rightarrow \gamma(S \times I) \subset M$ is a curve with $c(a) \in S_{t_{0}}$ and $c(b) \in S_{t_{1}}$, then we have $c(u)=\gamma(s(u), t(u))$ with $t(a)=t_{0}, t(b)=t_{1}$, and

$$
\left\|c^{\prime}(u)\right\|^{2}=\left\|d \gamma \cdot s^{\prime}(u)\right\|^{2}+t^{\prime}(u)^{2} \geq t^{\prime}(u)^{2}
$$

hence its length is

$$
L(c) \geq \int_{a}^{b}\left|t^{\prime}(u)\right| d u \geq|t(b)-t(a)| \geq\left|t_{0}-t_{1}\right| .
$$

Fig. 2.

In this case, all the quantities discussed above have geometric meanings. The Jacobi fields $J(t)=d \gamma_{(s, t)}(x, 0)=d \phi_{t} x$ for $x \in T_{s} S$ measure the change of the metric of $S_{t}=\phi_{t}(S)$ when $t$ is changed; in fact, $\|J(t)\| /\left\|J\left(t_{0}\right)\right\|$ is the length distortion between $S_{t}$ and $S$. Moreover, $A=D V$, restricted to the hypersurface $S_{t}$, is the shape operator of $S_{t}$ since $V \mid S_{t}$ is a unit normal vector field on $S_{t}$. Its eigenvalues are called principal curvatures, their average the mean curvature of $S_{t}$. Since Equation (2.7) is nonlinear, $A(s, t)$ can develop singularities which are called focal points of $S$. Let us see some examples.

Example 2.3 Let $S_{t}=\partial B_{t}(p)$, where $B_{t}(p)=\{x \in M:|x, p|<t\}$ is the Riemannian ball. 
Then $V$ is radial and

$$
A(t) \sim \frac{1}{t} I \quad \text { as } t \rightarrow 0
$$

because a Riemannian manifold behaves as a Euclidian space for $t \rightarrow 0$.

Example 2.4 ([9]) Let us suppose that $R_{V}=k I, k \in \mathbb{R}$, that is $M$ has constant curvature. Moreover let us suppose that $A=a I$, where $a$ is a real function defined on $M$ (A is the second fundamental form of a family of umbilical hypersurfaces). In this case equation (2.7) becomes:

$$
a^{\prime}+a^{2}+k=0
$$

If $k>0$, then $M$ is a sphere (if it is assumed to be complete and simply connected). The solutions are given by

$$
a(t)=\sqrt{k} \cot \left(\sqrt{k}\left(t-t_{0}\right)\right) .
$$

This corresponds to the fact that there is (up to congruence) only one equidistant family of umbilical hypersurfaces in the sphere, namlely concentric Riemannian spheres (latitude circles).

Fig. 3.

If $k=0$, then $M$ is a Euclidean space and the solutions are

$$
a(t)=\frac{1}{\left(t-t_{0}\right)}, \quad a(t)=0
$$


Fig. 4.

These solutions correspond to the three umbilical parallel hypersurface families in euclidean space: concentric spheres with increasing $\left(t>t_{0}\right)$ or decreasing $\left(t<t_{0}\right)$ radii and parallel hyperplanes.

Finally, if $k<0$, the space $M$ is hyperbolic. The solutions are given by

$$
a(t)=\sqrt{|k|} \operatorname{coth}\left(\sqrt{|k|}\left(t-t_{0}\right), \quad a(t)=\sqrt{|k|} \tanh \left(\sqrt{|k|}\left(t-t_{0}\right), \quad a(t)= \pm \sqrt{|k|} .\right.\right.
$$

These solutions correspond to the five families of equidistant hypersurfaces in the hyperbolic space: Concentric spheres with increasing $\left(t>t_{0}\right)$ or decreasing $\left(t<t_{0}\right)$ radii, hypersurfaces which are parallel to an $(n-1)$-dimensional hyperbolic subspace, and expanding $\left(t>t_{0}\right)$ or contracting $\left(t<t_{0}\right)$ horospheres.

Fig. 5. 


\section{Comparison theory.}

We want to derive a comparison theorem for solutions of the Riccati equation $A^{\prime}+A^{2}+R_{V}=0$ (cf. 2.7). Fixing an integral curve $\gamma$ of $V$ (which is a geodesic) and identifying all tangent spaces $T_{\gamma(t)} M$ by parallel displacement (i.e. via an orthonormal basis $\left(E_{i}(t)\right)$ of vector fields along $\gamma$ which are parallel, i.e. $\left.E_{i}^{\prime}=0\right)$, we consider $A(t)$ as a self adjoint endomorphism on a single vector space $E=T_{\gamma(0)} M$. More generally, let $E$ be a finite-dimensional real vector space with euclidean inner product $\langle$,$\rangle . The$ space $S(E)$ of self adjoint endomorphisms inherits the inner product

$$
\langle A, B\rangle=\operatorname{trace}(A \cdot B)
$$

for $A, B \in S(E)$. We get a partial ordering $\leq$ on $S(E)$ by putting $A \leq B$ if $\langle A x, x\rangle \leq$ $\langle B x, x\rangle$ for every $x \in E$.

Theorem 3.1 (cf. [14], [9]) Let $R_{1}, R_{2}: \mathbb{R} \rightarrow S(E)$ be smooth with $R_{1} \geq R_{2}$. For $i \in\{1,2\}$ let $A_{i}:\left[t_{0}, t_{i}\right) \rightarrow S(E)$ be a solution of

$$
A_{i}^{\prime}+A_{i}^{2}+R_{i}=0
$$

with maximal $t_{i} \in\left(t_{0}, \infty\right]$. Assume that $A_{1}\left(t_{0}\right) \leq A_{2}\left(t_{0}\right)$. Then $t_{1} \leq t_{2}$ and $A_{1}(t) \leq$ $A_{2}(t)$ on $\left(t_{0}, t_{1}\right)$.

Proof. Let $U=A_{2}-A_{1}$; then $U\left(t_{0}\right) \geq 0$ and

$$
U^{\prime}=A_{2}^{\prime}-A_{1}^{\prime}=A_{1}^{2}-A_{2}^{2}+R_{1}-R_{2}
$$

We define $S=R_{1}-R_{2} \geq 0$ and $X=-\frac{1}{2}\left(A_{1}+A_{2}\right)$; the equation (3.3) takes the form

$$
U^{\prime}=X U+U X+S \text {. }
$$

We solve (3.4) by the variation of constant method (see [14], pag. 211, Remark 1). Let $t^{\prime}=\min \left\{t_{1}, t_{2}\right\}$ and $g:\left(t_{0}, t^{\prime}\right) \rightarrow S(E)$ be a non-singular solution of the homogeneous equation

$$
g^{\prime}=X g
$$

Now a solution $U$ of (3.4) is obtained as

$$
U=g \cdot V \cdot g^{T}
$$

where $V$ verifies

$$
V^{\prime}=g^{-1} \cdot S \cdot\left(g^{-1}\right)^{T} .
$$

¿From $S \geq 0$ we get $V^{\prime} \geq 0$; this, combined with $V(0) \geq 0$, implies that $V \geq 0$ and hence $U \geq 0$. Thus $A_{1} \leq A_{2}$ on $\left(t_{0}, t^{\prime}\right)$. Since $A_{i}^{\prime}$ is bounded from above, a singularity can only be negative (going to $-\infty$ ). So $A_{1} \leq A_{2}$ implies $t^{\prime}=t_{1} \leq t_{2}$. 
Remark 3.2 Theorem 3.1 still holds if $A_{1}, A_{2}$ are singular at $t_{0}$, but $U=A_{2}-A_{1}$ has a continuous extension to 0 with $U(0) \geq 0$. See [14] for the proof. A similar argument also shows that $t_{1}<t_{2}$ if $A_{1}\left(t_{0}\right)<A_{2}\left(t_{0}\right)$; for a different proof of this fact see [11], Lemma 3.1.

The geometric interpretation of Theorem 3.1 is: principal curvatures (i.e. eigenvalues of the shape operator) of equidistant hypersurfaces decrease faster on the space of larger curvature. In particular, this is true for Riemannian spheres, as follows by Remark 3.2).

Now we want to find a comparison theorem for equation (2.6). For $A \in S(E)$, denote by $\lambda_{-}(A)$ the lowest eigenvalue and by $\lambda_{+}(A)$ the highest eigenvalue of $A$.

Theorem 3.3 Let $A_{1}, A_{2}:\left(t_{0}, t^{\prime}\right) \rightarrow S(E)$ such that

$$
\lambda_{+}\left(A_{1}(t)\right) \leq \lambda_{-}\left(A_{2}(t)\right) \quad \text { everywhere. }
$$

Let $J_{1}, J_{2}:\left(t_{0}, t^{\prime}\right) \rightarrow E$ be nonzero solutions of $J_{i}^{\prime}=A_{i} \cdot J_{i}$. Then $\left\|J_{1}\right\| /\left\|J_{2}\right\|$ is monotoneously decreasing.

Moreover, if

$$
\lim _{t \searrow t_{0}} \frac{\left\|J_{1}\right\|}{\left\|J_{2}\right\|}(t)=1
$$

then $\left\|J_{1}\right\| \leq\left\|J_{2}\right\|$.

Equality holds at some $t \in\left(t_{0}, t^{\prime}\right)$ iff for $i=1,2$ we have $J_{i}=j \cdot v_{i}$ on $\left[t_{0}, t\right]$ for some constant vector $v_{i} \in E$ with $A v_{i}=\lambda \cdot v_{i}$ and $j^{\prime}=\lambda \cdot j$, where $\lambda=\lambda_{+}\left(A_{1}\right)=\lambda_{-}\left(A_{2}\right)$.

Proof. Since $\left\|J_{i}\right\|$ is smooth, we can consider

$$
\frac{\left\|J_{i}\right\|^{\prime}}{\left\|J_{i}\right\|}=\frac{<J_{i}^{\prime}, J_{i}>}{\left\|J_{i}\right\|^{2}}=\frac{<A_{i} J_{i}, J_{i}>}{<J_{i}, J_{i}>} \in\left[\lambda_{-}\left(A_{i}\right), \lambda_{+}\left(A_{i}\right)\right]
$$

so that

$$
\log \left(\left\|J_{1}\right\|\right)^{\prime}=\frac{\left\|J_{1}\right\|^{\prime}}{\left\|J_{1}\right\|} \leq \lambda_{+}\left(A_{1}\right) \leq \lambda_{-}\left(A_{2}\right) \leq \frac{\left\|J_{2}\right\|^{\prime}}{\left\|J_{2}\right\|}=\log \left(\left\|J_{2}\right\|\right)^{\prime}
$$

hence

$$
\left(\log \frac{\left\|J_{1}\right\|}{\left\|J_{2}\right\|}\right)^{\prime} \leq 0
$$

which implies that $\left\|J_{1}\right\| /\left\|J_{2}\right\|$ is monotoneously decreasing.

If $\left\|J_{1}\right\| /\left\|J_{2}\right\|$ has the same value 1 at $t_{0}$ and $t$, then $\left\|J_{1}\right\|=\left\|J_{2}\right\|$ on $\left[t_{0}, t\right]$ and we recieve $J_{i}^{\prime}=A_{i} J_{i}=\lambda J_{i}$ from which the conclusion follows. 
We consider the most important special cases due to Rauch and Berger (called Rauch I and Rauch II in [5]):

\section{Rauch I}

Suppose that $J_{i}$ for $i=1,2$ are solutions of $J_{i}^{\prime \prime}+R_{i} J_{i}=0$ with $\lambda_{-}\left(R_{1}\right) \geq \lambda_{+}\left(R_{2}\right)$ and

$$
J_{i}(0)=0,\left\|J_{1}^{\prime}(0)\right\|=\left\|J_{2}^{\prime}(0)\right\| .
$$

Then $\left\|J_{1}\right\| \leq\left\|J_{2}\right\|$ up to the first zero of $J_{1}$.

\section{Rauch II}

Suppose that $J_{i}$ for $i=1,2$ are solutions of $J_{i}^{\prime \prime}+R_{i} J_{i}=0$ with $\lambda_{-}\left(R_{1}\right) \geq \lambda_{+}\left(R_{2}\right)$ and

$$
J_{i}^{\prime}(0)=0,\left\|J_{1}(0)\right\|=\left\|J_{2}(0)\right\| .
$$

Then $\left\|J_{1}\right\| \leq\left\|J_{2}\right\|$ up to the first zero of $J_{1}$.

In fact we apply the theorems 3.1 and 3.3 where in the first case, $A_{i}(t) \sim t^{-1} I$ as $t \rightarrow 0$ and in the second case, $A_{i}(0)=0$.

Corollary 3.4 Let $M$ be a complete manifold with $K \geq 0, p_{0}, p_{1} \in M$ and $\gamma:[0,1] \rightarrow$ $M$ a shortest geodesic segment connecting $p_{0}$ and $p_{1}$. Let $X \perp \gamma^{\prime}$ be a parallel vector field along $\gamma$. Put $p_{s}(t)=\exp t X(s)$ for all $s \in[0,1]$. Then

$$
\left|p_{0}(t), p_{1}(t)\right| \leq\left|p_{0}, p_{1}\right|
$$

with equality for some $t>0$ only if $p_{0}, p_{1}, p_{1}(t), p_{0}(t)$ bound a flat totally geodesic rectangle.

Proof. We have

$$
\left|p_{0}(t), p_{1}(t)\right| \leq \int_{0}^{1}\left\|\frac{\partial}{\partial s} p_{s}(t)\right\| d t
$$

and $J_{s}(t)=\frac{\partial}{\partial s} p_{s}(t)$ is a Jacobi field along the geodesic $\gamma_{s}(t)=p_{s}(t)$ with $J_{s}^{\prime}(0)=0$. Thus comparing with the euclidean case we get from Rauch II that $\left\|J_{s}(t)\right\| \leq\left\|J_{s}(0)\right\|$ which shows the inequality. If we have equality at $t_{1}>0$, the equality discussion of Theorem 3.3 shows that $J_{s}$ is parallel along $\gamma_{s} \mid\left[0, t_{1}\right]$. Moreover, the curves $s \mapsto p_{s}(t)$ are shortest geodesics of constant length for $0 \leq t \leq t_{1}$. Thus the surface $p:(s, t) \mapsto p_{s}(t)$ is a flat rectangle in $M$ with

$$
\frac{D}{\partial s} \frac{\partial p}{\partial s}=\frac{D}{\partial t} \frac{\partial p}{\partial s}=\frac{D}{\partial t} \frac{\partial p}{\partial t}=0
$$

so it is also totally geodesic, i.e. covariant derivatives of vector fields tangent to $p$ remain tangent to $p$. 


\section{Average comparison theorems.}

Now we consider the trace of the Riccati equation $A^{\prime}+A^{2}+R_{V}=0$ for self adjoint $A$. Since trace and derivative commute, we get

$$
\operatorname{trace}(A)^{\prime}+\operatorname{trace}\left(A^{2}\right)+\operatorname{Ric}(V)=0
$$

This is unfortunately not a differential equation for trace $(A)$, because of the term $\operatorname{trace}\left(A^{2}\right)$. However, put

$$
a=\frac{\operatorname{trace}(A)}{n-1} .
$$

(Note that $A(V)=D_{V} V=0$, so we consider $A$ as an endomorphism on the $(n-1)$ dimensional subspace $E=V^{\perp}$ of the tangent space.) Then

$$
A=a I+A_{0},
$$

with $\operatorname{trace}\left(A_{0}\right)=0$, so $A_{0}$ and $I$ are perpendicular. Hence,

$$
\operatorname{trace}\left(A^{2}\right)=\|A\|^{2}=a^{2}\|I\|^{2}+\left\|A_{0}\right\|^{2}=(n-1) a^{2}+\left\|A_{0}\right\|^{2}
$$

and we get, from the trace equation (4.1):

$$
a^{\prime}+a^{2}+r=0
$$

with

$$
r=\frac{1}{n-1}\left(\left\|A_{0}\right\|^{2}+\operatorname{Ric}(V)\right) \geq \frac{1}{n-1} \operatorname{Ric}(V) .
$$

Geometric meaning: $a(t)$ is the mean curvature of $S_{t}$.

Theorem 4.1 Suppose that $A:\left[t_{0}, t_{1}\right) \rightarrow S(E)\left(t_{1} \leq+\infty\right.$ maximal $)$ is a solution of

$$
A^{\prime}+A^{2}+R=0
$$

where $R: \mathbb{R} \rightarrow S(E)$ is given; suppose that for some constant $k \in \mathbb{R}$ :

(1) $\operatorname{trace}(R) \geq(n-1) k$

(2) $\operatorname{trace}\left(A\left(t_{0}\right)\right) \leq(n-1) \bar{a}\left(t_{0}\right)$

where $\bar{a}:\left[t_{0}, t_{2}\right) \rightarrow \mathbb{R}$ is a solution of

$$
\bar{a}^{\prime}+\bar{a}^{2}+k=0
$$


with $t_{2} \leq+\infty$ maximal. Let

$$
a=\frac{\operatorname{trace}(A)}{n-1} .
$$

Then $t_{1} \leq t_{2}$ and $a(t) \leq \bar{a}(t)$ for $t \in\left[t_{0}, t_{1}\right)$.

Proof. Apply theorem 3.1 with $\left(R_{1}, A_{1}, R_{2}, A_{2}\right)$ replaced with $(r, a, k, \bar{a})$.

Remark 4.2 By Remark 3.2, the theorem remains true if $A(t) \sim \frac{1}{t-t_{0}} I$ and $\bar{a}$ is the solution of (4.4) with a pole at $t_{0}$, i.e. $\bar{a}=s^{\prime} / s$, where $s$ is the solution of

$$
s^{\prime \prime}+k s=0, \quad s\left(t_{0}\right)=0, \quad s^{\prime}\left(t_{0}\right)=1 .
$$

Next, let $J_{1}, \ldots, J_{n-1}$ be a basis of solutions of $J^{\prime}=A \cdot J$, and put

$$
j=\operatorname{det}\left(J_{1}, \ldots, J_{n-1}\right) \text {. }
$$

Since

$$
\left(J_{1} \wedge \ldots \wedge J_{n-1}\right)^{\prime}=\sum_{k=1}^{n-1} J_{1} \wedge \ldots \wedge A \cdot J_{k} \wedge \ldots \wedge J_{n-1}
$$

we get

$$
j^{\prime}=(n-1) a \cdot j .
$$

Geometrically, equation (4.6) says how the volume element of $S_{t}$, namely $\operatorname{det}\left(d \phi_{t}\right)$ (see page 9 of chapter 2), changes with $t$.

Theorem 4.3 Let $A:\left[t_{0}, t_{1}\right) \rightarrow S(V)$ be given with

$$
a \leq \bar{a},
$$

where $a=\frac{1}{n-1} \operatorname{trace}(A)$, and let $j$ be as above. Choose $\bar{j}$ such that

$$
\bar{j}^{\prime}=(n-1) \bar{a} \cdot \bar{j} .
$$

Then $j / \bar{j}$ is monotonously decreasing.

Proof. Apply theorem 3.3 with $\left(A_{1}, J_{1}, A_{2}, J_{2}\right)$ replaced with $((n-1) a, j,(n-1) \bar{a}, \bar{j})$. 


\section{Bishop - Gromov inequality}

Let $M$ be a complete connected Riemannian manifold. By the theorem of Hopf and Rinow (cf. [29]), any two points $p, q \in M$ can be connected by a shortest geodesic $\gamma$, i.e. $L(\gamma)=|p, q|$. Let $S_{p} M=\left\{v \in T_{p} M:\|v\|=1\right\}$ be the unit sphere in $T_{p} M$. For any $v \in S_{p} M$, we define

$$
\operatorname{cut}(v)=\max \left\{t:\left.\gamma_{v}\right|_{[0, t]} \text { is shortest }\right\}
$$

This defines a function cut $: S_{p} M \rightarrow(0, \infty]$, the cut locus distance, which is continuous (cf [5], p.94). Let

$$
C_{p}=\left\{t v: v \in S_{p} M, \quad t \leq \operatorname{cut}(v)\right\} .
$$

This is a closed subset of $T_{p} M$, and its boundary $\partial C_{p}$ (sometimes also $\exp _{p}\left(\partial C_{p}\right) \subset M$ ) is called the cut locus of the point $p$. It follows from this definition that

$$
B_{r}(p)=\exp _{p}\left(B_{r}(0)\right)=\exp _{p}\left(B_{r}(0) \cap C_{p}\right) \quad \forall r>0
$$

In fact, if we choose $q \in B_{r}(p)$, there exists a shortest geodesic $\gamma_{v}$ joining $p$ and $q$; the length of $\gamma_{v}$ should be $\leq \operatorname{cut}(v)$, hence $v \in C_{p}$ (theorem of Hopf - Rinow).

Example 5.1 On the unit sphere we have $\operatorname{cut}(v)=\pi$ for every $v$. In fact, in every direction, the geodesic is a meridian, hence it is shortest up to the opposite ("antipodal") point.

Example 5.2 On the cylinder $S^{1} \times \mathbb{R}$, we have $\operatorname{cut}(v)=\pi / \cos \alpha$ where $\alpha$ is the angle between $v$ and the $S^{1}$-direction.

Fig. 6 . 
There are two ways how a geodesic $\gamma=\gamma_{v}:[0, \infty) \rightarrow M\left(\right.$ where $v \in S_{p} M$ ) can cease to be shortest beyond the parameter $t_{0}=\operatorname{cut}(v)$ (cf. [5], p.93): Either there exists a nonzero Jacobi field $J$ along $\gamma$ which vanishes at 0 and $t_{0}$ - in this case, $\gamma\left(t_{0}\right)$ is called a conjugate point of $p$ (cf. Example 5.1), or there exists a second geodesic $\sigma \neq \gamma$ of the same length which also connects $p$ and $\gamma\left(t_{0}\right)$ (cf. Example 5.2). Hence $q=\gamma\left(t_{0}\right)$ is in the cut locus of $p=\gamma(0)$ iff $p$ is in the cut locus of $q$. Moreover, there are no conjugate points on $\gamma \mid[0, c u t(v))$. The conjugate points in turn are the singular values of the exponential map $\exp _{p}$; more precisely, we have:

Lemma 5.3 Let $J(t)$ be the Jacobi field along $\gamma_{v}$ defined by $J(0)=0, J^{\prime}(0)=w$. Then we have

$$
d\left(\exp _{p}\right)_{t v} \cdot t w=J(t)
$$

In particular, $d\left(\exp _{p}\right)_{t v}$ is singular if and only if $\exp _{p}(t v)$ is a conjugate point of $p$.

Proof. Let $w \in T_{v} T_{p} M \equiv T_{p} M$. Then we have

$$
d\left(\exp _{p}\right)_{v} \cdot w=\left.\frac{d}{d s}\right|_{s=0} \exp _{p}(v+s w)=\left.\frac{d}{d s}\right|_{s=0} \gamma_{v+s w}(1)
$$

If we let

$$
J(t)=\left.\frac{\partial}{\partial s}\right|_{s=0} \gamma_{v+s w}(t),
$$

then $J$ is the Jacobi field along $\gamma_{v}$ with initial conditions $J(0)=0$ and

$$
\begin{aligned}
J^{\prime}(0) & =\left.\left.\frac{D}{\partial t}\right|_{0} \frac{\partial}{\partial s}\right|_{0} \gamma_{v+s w}(t) \\
& =\left.\left.\frac{D}{\partial s}\right|_{0} \frac{\partial}{\partial t}\right|_{0} \gamma_{v+s w}(t) . \\
& =\left.\frac{D}{\partial s}\right|_{0}(v+s w)=w
\end{aligned}
$$

Therefore we get

$$
d\left(\exp _{p}\right)_{v} \cdot w=J(1)
$$

and generally

$$
d\left(\exp _{p}\right)_{t v} \cdot t w=J(t) .
$$


Remark 5.4 Consequently, on the interior of $C_{p}$, the exponential map $\exp _{p}$ is injective and regular, hence a diffeomorphism. Note that $\operatorname{Int}\left(C_{p}\right)$ is star-shape, thus it is contractive; hence also its image is contractive. But by Hopf-Rinow, the whole manifold $M$ is the image of $\exp _{p}: C_{p} \rightarrow M$, so the topology of $M$ is given by the image of the boundary $\partial C_{p}$.

After these preparations, we come to the main theorem of this section.

Theorem 5.5 Let us consider a manifold $M^{n}$ with Ricci curvature satisfying

$$
\frac{R i c}{n-1} \geq k
$$

Let $\bar{M}$ be the complete simply connected n-manifold with constant curvature $k$ (standard space of constant curvature $k$ ) and $\bar{B}_{r} \subset \bar{M}$ the ball of radius $r$ in $\bar{M}$. Then, for all $p \in M$, we have that

$$
\frac{\operatorname{Vol} B_{r}(p)}{\operatorname{Vol} \bar{B}_{r}} \searrow r
$$

i.e. this quotient is monotonely decresing with $r$. Moreover, for $r \rightarrow 0$, the quotient goes to one.

Corollary 5.6 For any two positive real numbers $R>r$ we have

$$
\frac{\operatorname{Vol} B_{R}(p)}{\operatorname{Vol} B_{r}(p)} \leq \frac{\operatorname{Vol} \bar{B}_{R}}{\operatorname{Vol} \bar{B}_{r}}
$$

Remark 5.7 Corollary 5.6 gives an upper bound for the growth of the metric balls in $M$. Moreover, if equality holds for some $r<R$, then $B_{R}(p)$ is isometric to $\bar{B}_{r}$ (this can be seen from the proof).

Proof of the theorem. By (5.3) we have

$$
\operatorname{Vol} B_{r}(p)=\int_{B_{r}(0) \cap C_{p}} \operatorname{det}\left(d\left(\exp _{p}\right)_{u}\right) d u .
$$

Passing to polar coordinates and denoting $r(v)=\min \{r, \operatorname{cut}(v)\}$, we get

$$
\operatorname{Vol} B_{r}(p)=\int_{S} \int_{0}^{r(v)} \operatorname{det}\left(d\left(\exp _{p}\right) t v\right) t^{n-1} d t d v
$$


where $S:=S_{1}(0) \subset T_{p} M$. If we consider a basis $e_{1}, \ldots, e_{n-1}$ of $v^{\perp} \subset T_{p} M$, then by Lemma 5.3,

$$
d\left(\exp _{p}\right)_{t v} e_{i}=\frac{1}{t} d\left(\exp _{p}\right)_{t v} t e_{i}=\frac{1}{t} J_{i}(t)
$$

where $J_{i}$ is the Jacobi field along $\gamma_{v}$ with $J_{i}(0)=0$ and $J_{i}^{\prime}(0)=e_{i}$. Hence

$$
\operatorname{det}\left(d\left(\exp _{p}\right)_{t v}\right)=\frac{1}{t^{n-1}} \operatorname{det}\left(J_{1}(t), \ldots, J_{n-1}(t)\right),
$$

and equation (5.10) becomes

$$
\operatorname{Vol} B_{r}(p)=\int_{S} \int_{0}^{r(v)} j_{v}(t) d t d v
$$

where

$$
j_{v}(t)=\operatorname{det}\left(J_{1}(t), \ldots, J_{n-1}(t)\right) .
$$

If we put $j_{v}(t)=0$ for $t>\operatorname{cut}(v)$, then by the comparison theorem 4.3 we get

$$
\left(j_{v} / \bar{j}\right) \searrow
$$

on $[0, r]$ and hence

$$
q:=\frac{1}{\operatorname{Vol}(S)} \int_{S}\left(j_{v} / \bar{j}\right) d v
$$

is still monotone. Moreover,

$$
\operatorname{Vol} \bar{B}_{r}=\int_{S} \int_{0}^{r} \bar{j}(t) d t d v=\operatorname{Vol}(S) \int_{0}^{r} \bar{j}(t) d t
$$

Therefore we have that

$$
\frac{\operatorname{Vol}_{r}(p)}{\operatorname{Vol} \bar{B}_{r}}=\frac{\int_{0}^{r} q(t) \bar{j}(t) d t}{\int_{0}^{r} \bar{j}(t) d t}
$$

is a monotone decreasing function in $r$, because the mean of a monotone function on growing intervals is still monotone.

If $r \rightarrow 0$, both volumes approximate the euclidean ball volume, hence the quotient goes to one. 


\section{Toponogov's Triangle Comparison Theorem}

Let us fix $o \in M$ and let $\rho=|o, \cdot|$. We already know that near $o$, precisely in $\exp _{o}\left(\operatorname{Int}\left(C_{o}\right) \backslash\{0\}\right), \rho$ is a $C^{\infty}$ function and

$$
\rho\left(\exp _{o}(v)\right)=\|v\|
$$

Let us consider the unit radial field $V=\nabla \rho$. Then $S_{r}=\partial B_{r}(o)$ is a family of equidistant hypersurfaces, as in chapter 2.

Suppose that the sectional curvature $K$ of $M$ is $\geq k$. If $\tilde{M}$ is the standard space of sectional curvature $k$, then, by the comparison theorem 3.1, we get

$$
A \leq \tilde{A}=\frac{s^{\prime}}{s} I
$$

where $s$ is a solution of $s^{\prime \prime}+k s=0$ with initial dates $s(0)=0, s^{\prime}(0)=1$, and $A=$ $D V=D \nabla \rho$ is the Hessian of $\rho$. (Recall from Example 2.4 that $a=s^{\prime} / s$ is the (unique) solution of the equation $a^{\prime}+a^{2}+k=0$ with a pole at $t=0$.)

Therefore,

$$
\left.D \nabla \rho\right|_{V^{\perp}} \leq \frac{s^{\prime}}{s} I
$$

while

$$
\left.D \nabla \rho\right|_{\mathbb{R} V}=0,
$$

because $\rho$ grows linearly along the integral curves of $V$. Analogous relations hold for $\tilde{\rho}$ :

$$
\begin{gathered}
\left.D \nabla \tilde{\rho}\right|_{V^{\perp}}=\frac{s^{\prime}}{s} I \\
\left.D \nabla \tilde{\rho}\right|_{\mathbb{R} V}=0
\end{gathered}
$$

Now we want to find a unique estimate for the whole Hessian. To get this we modify $\rho$ (and analogously $\tilde{\rho}$ ) suitably: Consider $\sigma=f \circ \rho$, where $f: \mathbb{R} \rightarrow \mathbb{R}$ is a function yet to be determined. Then

$$
D \nabla(f \circ \rho)=D\left(\left(f^{\prime} \circ \rho\right) \nabla \rho\right)=f^{\prime \prime}(\rho) d \rho \cdot \nabla \rho+f^{\prime}(\rho) D \nabla \rho .
$$

On $V^{\perp}$ we have $f^{\prime \prime}(\rho) d \rho \cdot \nabla \rho=0$ and $D \nabla \rho \leq\left(s^{\prime} / s\right) I$; while $f^{\prime \prime}(\rho) d \rho \cdot \nabla \rho=f^{\prime \prime}(\rho) I$ and $D \nabla \rho=0$ on $\mathbb{R} \cdot V$. If we choose $f$ as a principal function of $s$, i.e. $f^{\prime}=s$, then we get

$$
f^{\prime \prime}(\rho)=-k f(\rho)+C,
$$

hence (6.3) and (6.4) give

$$
D \nabla \sigma \leq-k \sigma I+C
$$


where $C$ is some fixed constant. Analogously, for $\tilde{\sigma}=f \circ \tilde{\rho}$, we get from (6.5) and (6.6)

$$
D \nabla \tilde{\sigma}=-k \tilde{\sigma} I+C
$$

Theorem 6.1 (Toponogov's triangle comparison theorem) [18], [5], [24]

Let $M$ be a complete Riemannian manifold with sectional curvature $K \geq k$. Let $\tilde{M}$ be the standard space of constant curvature $k$. Let $p_{0}, p_{1}, o \in M$, and choose corresponding points $\tilde{p}_{0}, \tilde{p}_{1}, \tilde{o} \in \tilde{M}$. Let $\gamma$ be a geodesic from $p_{0}$ to $p_{1}$, and $\beta_{i}$ a shortest geodesic from $p_{i}$ to $o, i=0,1$, all parametrized by arc length, and let $\tilde{\gamma}, \tilde{\beta}_{i}$ be the corresponding curves in $\tilde{M}$, with $L(\gamma)=L(\tilde{\gamma})=L$ and $L\left(\beta_{i}\right)=L\left(\tilde{\beta}_{i}\right)$. Let us suppose that all the lengths are smaller than $\pi / \sqrt{k}$, if $k>0$. Then we have

$$
|o, \gamma(t)| \leq|\tilde{o}, \tilde{\gamma}(t)| \quad \forall t \in[0, L]
$$

Fig. 7.

Remark 6.2 The hypothesis made on lengths (when $k>0$ ) implies that the geodesics in $\tilde{M}$ are shortest.

Corollary 6.3 Let $\alpha_{0}=L\left(\beta_{0}^{\prime}, \gamma^{\prime}(0)\right), \alpha_{1}=L\left(\beta_{1}^{\prime},-\gamma^{\prime}(L)\right)$ and let $\tilde{\alpha}_{0}, \tilde{\alpha}_{1}$ the corresponding angles in $\tilde{M}$. Then

$$
\alpha_{i} \geq \tilde{\alpha}_{i}
$$

Proof of the corollary. Let us suppose, by contradiction, that $\alpha_{0}<\tilde{\alpha}_{0}$. Suppose first that $p_{0}$ is not in the cut locus of $o$. Then $\exp _{o}$ is invertible near $p_{0}$ (cf. Lemma 5.3). 
Let $\beta_{t}$ be the shortest geodesic joining $o$ to $\gamma(t)$; the corresponding one $\tilde{\beta}_{t}$ is a shortest geodesic in $\tilde{M}$ (for $t$ close to 0 ), hence

$$
\begin{aligned}
& L\left(\beta_{t}\right) \geq|o, \gamma(t)|, \\
& L\left(\tilde{\beta}_{t}\right)=|\tilde{o}, \tilde{\gamma}(t)| .
\end{aligned}
$$

We have

$$
\begin{aligned}
& L\left(\tilde{\beta}_{t}\right)=\left|o, p_{0}\right|+\left.t \frac{d}{d t}\right|_{0} L\left(\tilde{\beta}_{t}\right)+O\left(t^{2}\right) \\
& L\left(\beta_{t}\right)=\left|o, p_{0}\right|+\left.t \frac{d}{d t}\right|_{0} L\left(\beta_{t}\right)+O\left(t^{2}\right)
\end{aligned}
$$

and, by the first variation formula for curves (cf. [5], p.5), we get

$$
\begin{gathered}
\left.\frac{d}{d t}\right|_{0} L\left(\beta_{t}\right)=-<\gamma^{\prime}(0), \beta_{0}^{\prime}(0)> \\
\left.\frac{d}{d t}\right|_{0} L\left(\tilde{\beta}_{t}\right)=-<\tilde{\gamma}^{\prime}(0), \tilde{\beta}_{0}^{\prime}(0)>.
\end{gathered}
$$

Since we supposed $\alpha_{0}<\tilde{\alpha}_{0}$, for small $t$ we get $L\left(\gamma_{t}\right)<L\left(\tilde{\gamma}_{t}\right)$, which implies

$$
|o, \gamma(t)| \leq L\left(\gamma_{t}\right)<L\left(\tilde{\gamma}_{t}\right)=|\tilde{o}, \tilde{\gamma}(t)|
$$

Thus, by Toponogov's theorem, we get a contradiction.

If $p_{0}$ happens to be a cut locus point of $o$, we choose $o_{\varepsilon}=\beta_{0}(\varepsilon)$ on $\beta_{0}$ close to $o$. Then certainly $p_{0}$ is not in the cut locus of $o_{\varepsilon}$. Now we put $\beta_{t}$ the broken geodesic $\beta \mid[0, \varepsilon] \cup \beta_{\varepsilon, t}$ where $\beta_{\varepsilon, t}$ denotes the shortest geodesic from $o_{\varepsilon}$ to $\gamma(t)$, and the same argument holds.

Proof of theorem 6.1

Let us define $\rho=|o, \cdot|, \tilde{\rho}=|\tilde{o}, \cdot|$, and $\sigma=f \circ \rho, \tilde{\sigma}=f \circ \tilde{\rho}$. Consider the function

$$
\delta=\sigma \circ \gamma-\tilde{\sigma} \circ \tilde{\gamma}
$$

Hence we have to prove that

$$
\delta \geq 0 \quad \text { on }[0, L]
$$


Fig. 8 .

We prove $(6.15)$ by contradiction. Suppose that there is $t \in[0, L]$ such that $\delta(t)<0$, and let $m=\min _{[0, L]} \delta(t)<0$.

We choose $k^{\prime}>k$ sufficiently close to $k$ and $\tau>0$ such that

$$
L<\frac{\pi}{\sqrt{k^{\prime}}}-\tau \text {. }
$$

It is easy to find a solution $a_{0}$ of the equation $a_{0}^{\prime \prime}+k^{\prime} a_{0}=0$, with $a_{0}(-\tau)=0$ and $\left.a_{0}\right|_{[0, L]} \leq m$. Then there exists $\lambda>0$ such that $a=\lambda a_{0}$ satisfies the following properties:

1. $a \leq \delta$

2. $a\left(t_{0}\right)=\delta\left(t_{0}\right)$ for some $t_{0} \in(0, L)$.

Case 1: $\gamma\left(t_{0}\right)$ is not a cut locus point of $o$. Thus $\delta$ is of class $C^{\infty}$ in a neighborhood of $t_{0}$ and

$$
(\sigma \circ \gamma)^{\prime \prime}=<D_{\gamma^{\prime}} \nabla \sigma, \gamma^{\prime}>\leq-k(\sigma \circ \gamma)+C,
$$

where the inequality follows from (6.8). By eqution (6.9) we get

$$
(\tilde{\sigma} \circ \tilde{\gamma})^{\prime \prime}=<D_{\tilde{\gamma}^{\prime}} \nabla \tilde{\sigma}, \tilde{\gamma}^{\prime}>=-k(\tilde{\sigma} \circ \tilde{\gamma})+C .
$$

Hence

$$
\delta^{\prime \prime} \leq-k \delta .
$$

On the other hand $a^{\prime \prime}=-k^{\prime} a$. Moreover, in $t_{0}$ we have $\delta\left(t_{0}\right)=a\left(t_{0}\right)<0$, which implies

$$
(\delta-a)^{\prime \prime}\left(t_{0}\right) \leq \delta\left(t_{0}\right)\left(k^{\prime}-k\right)<0 .
$$

This is a contradiction because $\delta-a$ takes a minimum at $t_{0}$. 
Case 2: $\gamma\left(t_{0}\right)$ is a cut locus point of $o$. Let $\beta$ be a shortest geodesic from $o$ to $\gamma\left(t_{0}\right)$. We choose $o_{\varepsilon}$ on $\beta$ close to $o$, say $\left|o_{\varepsilon}, o\right|=\varepsilon$. Then we replace $\rho$ by $\rho_{\varepsilon}(x):=\left|x, o_{\varepsilon}\right|+\left|o_{\varepsilon}, o\right|$. By triangle inequality,

$$
\rho_{\varepsilon}(x) \geq \rho(x),
$$

and equality holds at $x=\gamma\left(t_{0}\right)$. In other words, $\rho_{\varepsilon}$ is an upper support function of $\rho$ at $\gamma\left(t_{0}\right)$. Since $\beta$ is shortest from $o$ to $\gamma\left(t_{0}\right), o_{\varepsilon}$ is not a cut point of $\gamma\left(t_{0}\right)$, and therefore, $\gamma\left(t_{0}\right)$ is not a cut point of $o_{\varepsilon}$ (cf. Ch.5). Putting $\sigma_{\varepsilon}=f \circ \rho_{\varepsilon}$, we get the same estimates as in Case 1 for $\sigma_{\varepsilon}$ in place of $\sigma$, up to a small error which goes to zero as $\varepsilon \rightarrow 0$ :

$$
\left(\sigma_{\varepsilon} \circ \gamma\right)^{\prime \prime} \leq-k\left(\sigma_{\varepsilon} \circ \gamma\right)+C+\text { error }
$$

Now $\sigma_{\varepsilon}$ is an upper support function of $\sigma$ at $\gamma\left(t_{0}\right)$ as $f$ is monotoneously increasing. Hence $\delta_{\varepsilon}-a$ is an upper support function of $\delta-a$ at $t_{0}$ where $\delta_{\varepsilon}=\sigma_{\varepsilon}-\tilde{\sigma}$. Thus it also takes a minimum at $t_{0}$. But this is a contradiction, because $\left(\delta_{\varepsilon}-a\right)^{\prime \prime}\left(t_{0}\right)<0$ by $(6.20)$.

Remark 6.4 The above proof is essentially due to Karcher ([24]). Recently, M. Kürzel ([27]) extended this proof to the case where curvature bounds are given which depend radially on the point (rather than being constant). 


\section{Number of generators and growth of the fundamental group}

Let $M$ be a complete Riemannian manifold and $\hat{M}$ its universal covering. The fundamental group $\pi_{1}(M)$ will be viewed as group of deck transformations acting on $\hat{M}$. In other words, $M$ is the orbit space of a discrete group $\Gamma \cong \pi_{1}(M)$ of isometries of $\hat{M}$ acting freely on $\hat{M}$, i.e. if $g \in \Gamma$ with $g(p)=p$ for some $p \in M$, then $g=1$.

Remark 7.1 The fundamental group of any compact Riemannian manifold $M$ is finitely generated.

Proof. There exists a compact fundamental domain $F$ (see definition below) for the action of $\Gamma$ on $\hat{M}$; e.g. one may take the so called Dirichlet fundamental domain

$$
F=\{x \in \hat{M} ; \quad|x, o| \leq|x, g o| \forall g \in \Gamma\} .
$$

We say that $g \in \Gamma$ is small if $g F \cap F \neq \emptyset$, i.e. if the fundamental domains $F$ and $g F$ are neighbors. If $d(F)$ denotes the diameter of $F$, i.e. the largest possible distance within $F$, then $g F \subset B_{2 d(F)}(o)$ for all small $g$, for some fixed $o \in F$. Since the subsets $g(\operatorname{Int}(F))$ are all disjoint with equal volume, there can be only finitely many of them in this ball, hence there exist only finitely many small $g$. We claim that they form a set of generators. In fact, let $g \in \Gamma$ arbitrary. Choose a geodesic segment $\gamma$ from $o$ to go. Then $\gamma$ is covered by finitely many fundamental domains $g_{0} F, \ldots, g_{N} F$ where $g_{0}=1$ and $g_{N}=g$, and $g_{i-1} F, g_{i} F$ are neighbors. Thus $g_{i}^{-1} g_{i-1}$ is small, and hence $g$ is a composition of small group elements.

Definition 7.2 A closed subset $F \subset \hat{M}$ is a fundamental domain for a group $\Gamma$ acting on $\hat{M}$ if

(a.) $\operatorname{Int}(F) \cap \operatorname{Int}(g F)=\emptyset \forall g \neq 1$;

(b.) $\Gamma \cdot F=\hat{M}$.

For a noncompact manifold $M$, the fundamental group may have infinitely many generators. The next theorem shows that this does not happen if $M$ has $K \geq 0$; in fact, there is an a-priori bound on the number of generators, i.e. the cardinality of a suitably chosen set of generators:

Theorem 7.3 (Gromov 1978, cf [24])

There exists a number $c(n)$ such that:

(a) the number of generators for $\pi_{1}(M)$ is $\leq c(n)$ for any n-dimensional complete manifold $M$ with curvature $K \geq 0$.

(b) the number of generators for $\pi_{1}(M)$ is $\leq c(n)^{1+k D}$ for any n-dimensional compact manifold $M$ with curvature $K \geq-k^{2}$ and diameter bounded, $\operatorname{diam}(M) \leq D$. 
Proof. We prove only part a); the second part is similar, but more technical (see Remark at the end of the proof).

We define a "norm" in $\Gamma$ as follows:

$$
|g|=|p, g(p)|
$$

for some fixed $p \in \hat{M}$. There exists $g_{1} \in \Gamma \backslash\{1\}$ with $\left|g_{1}\right|$ minimal (not necessarily unique). By induction, we can construct a sequence $\left(g_{j}\right)$ : given $g_{1}, \ldots, g_{k}$, we define

$$
\Gamma_{k}=\left\langle g_{1}, \ldots, g_{k}\right\rangle \subset \Gamma
$$

and choose $g_{k+1} \in \Gamma \backslash \Gamma_{k}$ such that $\left|g_{k+1}\right|$ has minimum norm in $\Gamma \backslash \Gamma_{k}$. To finish the proof, we only have to show

Claim: $\Gamma_{k}=\Gamma$ for some $k \leq c_{0}(n):=2 \sqrt{5}^{n}$.

Proof of the claim: for $j>i$ we have $\left|g_{j}\right| \geq\left|g_{i}\right|$, and moreover

$$
\left|g_{i}(p), g_{j}(p)\right|=\left|p, g_{i}^{-1} g_{j}(p)\right|=\left|g_{i}^{-1} g_{j}\right| \geq\left|g_{j}\right|
$$

since $g_{i}^{-1} g_{j} \in \Gamma \backslash \Gamma_{j-1}$ (otherwise $g_{i}^{-1} g_{j}, g_{i} \in \Gamma_{j-1}$ which would imply that $g_{j} \in \Gamma_{j-1}$ contradicting the choice of $\left.g_{j}\right)$. Now consider the triangle $p, p_{i}=g_{i}(p), p_{j}=g_{j}(p)$.

Fig. 9 .

Let $\gamma_{v_{i}}$ be a shortest geodesic from $p$ to $p_{i}$, and $\alpha_{i j}$ the angle between $v_{i}$ and $v_{j}$. The standard space $\tilde{M}$ of zero curvature is euclidean space $\mathbb{R}^{n}$. Considering the comparison triangle $\tilde{p}, \tilde{p}_{i}, \tilde{p}_{j}$ in $\tilde{M}$, we have $\tilde{\alpha}_{i j} \geq 60^{\circ}$ for the corresponding angle $\tilde{\alpha}_{i j}$ in $\tilde{p}$. (Note that $\tilde{\alpha}_{i j}$ is opposite to the largest edge in that triangle.) By Toponogov's theorem then

$$
\alpha_{i j} \geq 60^{\circ} \quad \forall i \neq j .
$$


Fig. 10.

There are at most $2 \sqrt{5}^{n}$ vectors that satisfy (7.1). Namely, for any two vectors $v_{i}$, $v_{j}$ of this kind, balls of radii $\frac{1}{2}$ are disjoint and their inner half balls are contained in $B_{\sqrt{5} / 2}(0)$, as the figure shows.

Thus, if there are $k$ such vectors, then

$$
\operatorname{Vol} B_{\sqrt{5} / 2}(0) \geq \frac{k}{2} \operatorname{Vol} B_{\frac{1}{2}}
$$

hence

$$
k \leq 2 \frac{\operatorname{Vol} B_{\frac{\sqrt{5}}{2}}}{\operatorname{Vol} B_{\frac{1}{2}}}=2 \sqrt{5}^{n} .
$$

This finishes the proof of the claim and of the theorem.

Remark 7.4 A much better (but more difficult) estimate was given by U.Abresch (cf. $[1])$.

Remark 7.5 In Case (b), we use comparison with a hyperbolic triangle (curvature $-k^{2}$ ) instead of a euclidean one. Since the side lengths are a priori bounded by the diameter bound $D$, this is not much difference. To see that such a bound is necessary, let $M$ be a compact surface of genus $g$ with constant negative curvature. Then $\pi_{1}\left(M_{g}\right)$ is generated by $2 g$ elements, hence is not bounded as $g \rightarrow \infty)$. Nevertheless the theorem holds, since either the curvature or the diameter are unbounded as $g \rightarrow \infty$.

Now let us assume that $M$ has Ric $\geq 0$ rather than $K \geq 0$. If $M$ is complete and noncompact, it is an open question whether the fundamental group is finitely generated. However, for any finitely generated subgroup, the growth of this group is only polynomial: 
Definition 7.6 Let $\Gamma$ be a finitely generated group and $G$ a finite set of generators of $\Gamma$ with $G=G^{-1}$ and $1 \in G$. We define the growth function $N(k)$ (depending on $\Gamma$ and $G)$ as follows:

$$
N(k)=\sharp\left\{g \in \Gamma \mid \exists g_{1}, \ldots, g_{k} \in G \text { such that } g=g_{1} \cdot \ldots \cdot g_{k}\right\} .
$$

So $N(k)$ is the number of group elements which can be written as a product of $k$ elements of $G$. The dependence of $N(k)$ on $G$ is easy to estimate: If $G^{\prime}$ is another such generating set, then there are numbers $p, q$ such that any element of $G$ can be expressed by $p$ elements of $G^{\prime}$ and each element of $G^{\prime}$ by $q$ elements of $G$. Thus we have

$$
N^{\prime}(k) \geq N(q k), \quad N(k) \geq N^{\prime}(p k) .
$$

Theorem 7.7 (Milnor '68, [30])

Let $M$ be a complete manifold with Ric $\geq 0$ and let $\Gamma \subset \pi_{1}(M)$ any finitely generated subgroup of the fundamental group. Then the growth of $\Gamma$ can be estimated by

$$
N(k)<c \cdot k^{n} .
$$

where the constant $c$ depends on $\hat{M}$ and the chosen set of generators of $\Gamma$.

Proof. Let $G$ be a set of generators as above; it has $N(1)$ elements. Fix a point $o \in \hat{M}$. For all $g \in \Gamma$, let $|g|=|o, g o|$. Put $R^{\prime}=\max \{|g| ; g \in G\}$. Choose some $r>0$ small enough, so that

$$
B_{r}(g o) \cap B_{r}(o)=\emptyset \quad \forall g \in \Gamma \backslash\{1\}
$$

Put $R=R^{\prime}+r$. Then the family of balls $\left\{B_{r}(g o) ; g \in G\right\}$ is disjoint and its union is contained in $B_{R}(o)$ so that

$$
\operatorname{Vol}\left(B_{R}(o)\right) \geq N(1) \cdot \operatorname{Vol}\left(B_{r}(o)\right)
$$

We can iterate this argument as follows: At the second step, we consider

$$
G^{2}:=\left\{g_{1} g_{2} ; g_{1}, g_{2} \in G\right\}
$$

with $\sharp\left(G^{2}\right)=N(2)$. Then all balls $B_{r}(g o)$ with $g \in G^{2}$ are disjoint and contained in $B_{2 R}(o)$ so that

$$
\operatorname{Vol}\left(B_{2 R}(o)\right) \geq N(2) \cdot \operatorname{Vol}\left(B_{r}(o)\right) .
$$

In general, we obtain that

$$
\operatorname{Vol}\left(B_{k R}(o)\right) \geq N(k) \cdot \operatorname{Vol}\left(B_{r}(o)\right) .
$$


Recall that we have the Bishop - Gromov inequality (cf. Corollary 5.6),

$$
\operatorname{Vol}\left(B_{k R}(o)\right) \leq \omega_{n} k^{n} R^{n}
$$

where $\omega_{n}$ denotes the volume of the euclidean unit ball, hence

$$
N(k) \leq\left\{\frac{\omega_{n} R^{n}}{\operatorname{Vol}\left(B_{r}(o)\right)}\right\} k^{n}
$$




\section{Gromov's estimate of the Betti numbers}

Homology is a main tool to measure the complexity of topology. Fix a field $\mathbf{F}$ and let $H_{q}(M)$ denote the $q$-th singular homology of $M$ with coefficients in $\mathbf{F}$. Further, let $H_{*}(M)=\oplus_{q \geq 0} H_{q}(M)$ be the total homology of $M$. The total Betti number of $M$ is given by

$$
b(M)=\operatorname{dim}_{\mathbf{F}} H_{*}(M) .
$$

Theorem 8.1 Gromov, 1980 (cf. [15], [1], [28])

There is a constant $C(n)$ such that:

(a.) any complete $n$-dimensional manifold $M$ with nonnegative curvature $K$ satisfies

$$
b(M) \leq C(n)
$$

(b.) any compact $n$-dimensional manifold $M$ with curvature $K \geq-k^{2}$, and bounded diameter, $\operatorname{diam}(M) \leq D$, satisfies

$$
b(M) \leq C(n)^{1+k D}
$$

We will give the proof of part (a.), following ideas of Abresch [1] and W.Meyer [28]. (Part (b.) is similar, cf. Remark 7.2.) The proof uses the estimates of BishopGromov and Toponogov. It can be viewed as an application of some sort of Morse theory for the distance function $\rho(x)=|o, x|$ where $o \in M$ is fixed. In ordinary Morse theory, one considers a smooth function $f: M \rightarrow \mathbb{R}$ with isolated critical points with nondegenerate Hessian ( $p$ critical means that $\nabla f(p)=0$ ), and one observes how the topology of $M^{c}=\{x \in M ; f(x)<c\}$ is changed as $c$ grows. There are two main facts in Morse theory (cf [29]):

(1.) If $M^{b} \backslash M^{a}$ contains no critical points, then $M^{b}$ and $M^{a}$ are diffeomorphic.

(2.) If $M^{b} \backslash M^{a}$ contains exactly one critical point $p$, then $M^{b}$ is homotopic to $M^{a}$ with a $k$-cell attached, where $k$ is the index of the Hessian of $f$ at $p$.

The distance function $\rho=|o|:, M \rightarrow \mathbb{R}$ is no longer smooth, but we still have the notions of critical and regular points:

Definition 8.2 A point $x \in M$ is called a regular point of $\rho$ if there exists $v \in T_{x} M$ such that

$$
\left\langle v, \gamma^{\prime}(0)\right\rangle<0
$$

for any shortest geodesic $\gamma$ from $x$ to $o$. Any such vextor $v$ is called gradientlike. 
A point $x \in M$ is a critical point for $\rho$ if it is non-regular, i.e. if for any $v \in T_{x} M$ there is a shortest geodesic $\gamma$ from $x$ to $o$ such that

$$
\left\langle v, \gamma^{\prime}(0)\right\rangle \geq 0
$$

Remark 8.3 These notions make sense also if the point $o$ is replaced by a closed subset $\Sigma \subset M$. This will be needed in Ch.10.

Fact (1.) is still valid: Since the set of initial vectors of shortest geodesics to $o$ is closed, the gradientlike vectors form an open subset of $T M$ and moreover a convex cone at any regular point. Thus we may cover the closure of $M^{b} \backslash M^{a}=B_{b}(o) \backslash B_{a}(o)$ by finitely many open sets with gradientlike vector fields and past them together using a partition of unity, thus getting a gradientlike vector field in a neighborhood of the closure of $B_{b}(o) \backslash B_{a}(o)$. This has the property that $\rho$ is strictly increasing along its integral curves. Hence, pushing along the integral curves, we may deform the bigger ball $B_{b}(o)$ into the smaller one $B_{a}(o)$. (See Lemma 10.9 for details.) We will use this in Lemma 8.10 below.

However, Fact (2.) has no meaning and has to be replaced by another idea: Large balls can be covered by a bounded number of small balls (Bishop-Gromov inequality), and the jump of the Betti number when passing from a small ball to a large ball can be controlled using Toponogov's theorem.

First of all, critical points of $\rho$ are not necessarily isolated, but still in some sense, we have to take only finitely many into account:

Lemma 8.4 Let $M$ be a complete manifold with nonnegative curvature. For any $L>1$ there exists a finite number $c(n, L)$ such that there are at most $c(n, L)$ critical points $\left\{q_{i}\right\}$ for $\rho$ satisfying

$$
\left|o, q_{i+1}\right| \geq L\left|o, q_{i}\right|
$$

E.g. for $L=2$ we have $c(n, 2)=2 \sqrt{5}^{n}$.

Proof. Let $\left(q_{1}, q_{2}, \ldots\right)$ be a maximal sequence satisfying (8.5). For $i<j$, let $\gamma$ be a shortest geodesic from $q_{i}$ to $q_{j}$ and put $v=\gamma^{\prime}(0)$. Since $q_{i}$ is critical, there is a shortest geodesic $c$ from $q_{i}$ to $o$ with the angle $\beta=\angle\left(c^{\prime}(0), v\right) \leq 90^{\circ}$. Applying Toponogov's theorem (Corollary 6.3 ) with the standard space $\tilde{M}=\mathbb{R}^{n}$, we get $\tilde{\beta} \leq 90^{\circ}$.

Consider first the limit case $\tilde{\beta}=90^{\circ}$. Let $\tilde{\alpha}$ be the angle in $\tilde{o}$. It follows that

$$
\cos (\tilde{\alpha})=\frac{\left|q_{i}, o\right|}{\left|q_{j}, o\right|} \leq \frac{1}{L} .
$$


Fig. 11.

Hence, if $\tilde{\beta} \leq 90^{\circ}$,

$$
\tilde{\alpha}_{o} \geq \arccos \left(\frac{1}{L}\right)=: \alpha_{0} .
$$

Now we apply Toponogov's theorem backwards for the angle at $o$, but this time we consider an arbitrary shortest geodesic from $q_{i}$ to $q_{j}$. Then for the angle $\alpha$ at $o$ we have

$$
\alpha \geq \tilde{\alpha} \geq \alpha_{0}
$$

It follows as in Ch.7 that there must be a finite number of such critical points. If $L=2$, we have $\alpha_{0}=60^{\circ}$ and hence $c(n, 2)=2 \sqrt{5}^{n}$ as in the proof of Theorem 7.3.

Corollary 8.5 Given a complete manifold $M$ with nonnegative curvature, all critical points are contained in a finite ball.

Since we will work with many metric balls in $M$, we agree on the following convention: If $B=B_{r}(p)$ be a fixed ball and $\lambda>0$, we put $\lambda B:=B_{\lambda r}(p)$. More generally, for any $q \in M$ we let $\lambda B(q):=B_{\lambda r}(q)$.

Definition 8.6 Let $A \subset C \subset M$. We define the content of $A$ in $C$ as the rank of the inclusion map on the homology level:

$$
\operatorname{cont}(A, C)=\operatorname{rk}\left(i_{*}: H_{*}(A) \rightarrow H_{*}(C)\right)
$$

Then we define the content of a metric ball $B$ as:

$$
\operatorname{cont}(B)=\operatorname{cont}(B, 5 B) \text {. }
$$


Essentially, the content measures the total Betti number of a subset. But it is better than the Betti number since it has a nice monotonicity property: Note that if $A \subset A^{\prime} \subset$ $B^{\prime} \subset B \subset M$ then

$$
\operatorname{cont}(A, B) \leq \operatorname{cont}\left(A^{\prime}, B^{\prime}\right)
$$

In the spirit of Morse theory, we will observe how the content of balls grows with the radius. A measure for the number of critical points which are still outside the ball and which will eventually increase the content is the corank. This definition involves also critical points of the distance function $\rho_{p}=|p$,$| for points p \in M$ different from $o$, called critical for $p$ for short.

Definition 8.7 Let $B=B_{r}(o)$ a ball in $M, r>0$. For $p \in M$, let $k(r, p)$ be the maximum number of critical points $q_{1}, \ldots, q_{k}$ for $p$ such that

1) $\left|p, q_{1}\right| \geq 3 \mathrm{Lr}$

2) $\left|p, q_{i+1}\right| \geq L\left|p, q_{i}\right|$.

By Lemma (8.3), $k(r, p) \leq c(L, n)$. Then we define the corank of $B$ as follows:

$$
\operatorname{corank}(B)=\inf \{k(r, p) \mid p \in 5 B\} .
$$

Not all balls really contribute to the topology, namely the compressible ones:

Definition 8.8 A ball $B \subset M$ is called compressible if there is $\tilde{B}=\frac{3}{5} B(q)$ for some $q \in 2 B$, and a diffeomorphism $\varphi: M \rightarrow M$ such that

$$
\left.\varphi\right|_{M \backslash 5 B}=i d
$$

and

$$
\varphi(B) \subset \tilde{B}
$$

For short: $B$ is compressible into $\tilde{B}$. Otherwise, the ball $B$ is called incompressible.

Lemma 8.9 Suppose that $B$ is compressible into $\tilde{B}$, with $\tilde{B}=\frac{3}{5} B(p)$, and $p \in 2 B$. Then

$$
\operatorname{cont}(\tilde{B}) \geq \operatorname{cont}(B)
$$

and

$$
\operatorname{corank}(\tilde{B}) \geq \operatorname{corank}(B)
$$

Proof. Let $\varphi$ be the diffeomorphism which compresses $B$ into $\tilde{B}$. From $B \approx \varphi(B)$ and

$$
\varphi(B) \subset \tilde{B} \subset 5 \tilde{B} \subset 5 B
$$


it follows that $\operatorname{cont}(\tilde{B}) \geq \operatorname{cont}(B)$.

To show the second relation, put $k=\operatorname{corank}(B)$. Let $p \subset 5 \tilde{B} \subset 5 B$. Then there are $m \geq k$ critical points $q_{1}, \ldots, q_{m}$ for $p$ with

$$
\left|p, q_{1}\right| \geq 3 L r \geq 3 L \cdot \frac{3}{5} r, \quad\left|p, q_{i+1}\right| \geq L\left|p, q_{i}\right| .
$$

Hence $k\left(\frac{3}{5} r, p\right) \geq m \geq k$ which $\operatorname{shows} \operatorname{corank}(\tilde{B}) \geq k$.

Lemma 8.10 If $B$ is incompressible, for any $p \in 2 B$ there is a critical point for $p$ in $5 \tilde{B} \backslash \tilde{B}$, where $\tilde{B}=\frac{3}{5} B(q)$.

Proof. Otherwise, we could deform $B \subset 5 \tilde{B}$ into $\tilde{B}$ while keeping $M \backslash 5 B$ fixed, so $B$ would be compressible.

Lemma 8.11 Let $B$ be incompressible, and put $\tilde{B}=\lambda B(\tilde{p})$ for some $\lambda \leq \frac{1}{5 L}$ and $\tilde{p} \in \frac{3}{2} B$. Then

$$
\operatorname{corank}(\tilde{B}) \geq \operatorname{corank}(B)+1 .
$$

Proof. Let $\tilde{p} \in \frac{3}{2} B$ and $p \in 5 \tilde{B} \subset 2 B$. By the previous lemma, there is a critical point $q_{0}$ for $p$ in $3 B(p) \backslash \frac{3}{5} B(p)$, so we have

$$
3 r \geq\left|p, q_{0}\right| \geq \frac{3}{5} r \geq 3 L \lambda r .
$$

Now let $k=\operatorname{corank}(B)$. Then there are $m \geq k$ critical points $q_{1}, \ldots, q_{m}$ for $p$ with

$$
\left|p, q_{1}\right| \geq 3 L r, \quad\left|p, q_{i+1}\right| \geq L\left|p, q_{i}\right| .
$$

Thus by (8.13),

$$
\left|p, q_{1}\right| \geq L\left|p, q_{0}\right|, \quad\left|p, q_{0}\right| \geq 3 L \lambda r .
$$

Now (8.14) and (8.15) show

$$
k(\lambda r, p) \geq m+1 \geq k+1 .
$$

Since $p \in 5 \tilde{B}$ was arbitrary, we get

$$
\operatorname{corank}(\tilde{B}) \geq k+1 .
$$




\section{Proof of theorem 8.1}

Let $K=\max \{\operatorname{corank}(B) \mid B$ ball in $M\}$. By Lemma $8.4, K \leq c(n, L)$, and by Corollary 8.5 , for a large enough ball $B$, there exists an isotopy of $M$ that carries $M$ into $B$, hence

$$
\operatorname{cont}(B)=\operatorname{cont}(M, M)=b(M) .
$$

Gromov's theorem now follows if we prove that the content of any metric ball is bounded by a constant. This is done in 5 steps:

Step 1. If $\operatorname{corank}(B)=K$ then $\operatorname{cont}(B)=1$.

Proof: Let $p_{0}:=p$. By Lemma $8.11, B$ is compressible into $B_{1}=\frac{3}{5} B\left(p_{1}\right)$ for some $p_{1} \in 2 B$ (otherwise, we could increase the corank). Hence, $\left|p_{0}, p_{1}\right|<2 r$. By Lemma $8.9, \operatorname{corank}\left(B_{1}\right)=K$ and $\operatorname{cont}\left(B_{1}\right) \geq \operatorname{cont}(B)$. Repeating the argument, we compress $B_{1}$ (and hence $B$ ) into $B_{2}=\frac{3}{5} B_{1}\left(p_{2}\right)=\left(\frac{3}{5}\right)^{2} B\left(p_{2}\right)$ for some $p_{2} \in 2 B_{1}$. Hence $\left|p_{1}, p_{2}\right|<$ $\frac{3}{5} \cdot 2 r$. After $N$ steps, we have compressed $B$ into $B_{N}=\left(\frac{3}{5}\right)^{N} B\left(p_{N}\right)$ with $\left|p_{i}, p_{i+1}\right|<$ $\left(\frac{3}{5}\right)^{i} \cdot 2 r$ for $i=0, \ldots, N$. So we have for all $q \in B_{N}$ :

$$
|p, q|<\sum_{i=0}^{N}\left(\frac{3}{5}\right)^{i} \cdot 2 r<5 r .
$$

Thus $B_{N} \subset 5 B$. Since the cut locus distance (injectivity radius) is bounded below on the closure of $B$ (by compactness), we find some $N$ such that $\left(\frac{3}{5}\right)^{N} r$ is smaller than this bound which implies that $B_{N}$ is diffeormorphic to a euclidean ball and hence contractible. So, $\operatorname{cont}\left(B_{N}\right)=1$ since $b\left(B_{N}\right)=1$. On the other hand,

$$
\operatorname{cont}\left(B_{N}\right) \geq \operatorname{cont}\left(B_{N-1}\right) \geq \ldots \geq \operatorname{cont}(B)
$$

which proves $\operatorname{cont}(B)=1$.

Step 2. Each ball $B$ with $\operatorname{cont}(B) \geq 2$ is either incompressible or it contains an incompressible ball $\tilde{B}$ with $\operatorname{cont}(\tilde{B}) \geq \operatorname{cont}(B)$ and $\operatorname{corank}(\tilde{B}) \geq \operatorname{corank}(B)$.

Proof. Otherwise, we could use the process of Step 1 to show that $\operatorname{cont}(B)=1$. satisfies

Step 3. If $B$ is incompressible, then any ball $\tilde{B}=\lambda B(p)$ with $\lambda \leq \frac{1}{5 L}$ and $p \in \frac{3}{2} B$

$$
\operatorname{corank}(\tilde{B}) \geq \operatorname{corank}(B)+1 \text {. }
$$

\section{Proof. Cf. Lemma 8.11.}

Step 4. There is a number $\chi \leq(2 L)^{n(n+1)} 10^{n(n+1)(n+2)}$ with the following property: Suppose that any ball $\tilde{B}$ with $\operatorname{corank}(\tilde{B}) \geq k$ has $\operatorname{cont}(\tilde{B}) \leq a_{k}$. Then for any ball $B$ with $\operatorname{corank}(B) \geq k-1$ we have

$$
\operatorname{cont}(B) \leq \chi \cdot a_{k}
$$


Proof. By Step 2 we may assume that $B$ is incompressible. Let $\lambda=1 /\left(5 L \cdot 10^{n+1}\right)$. We cover $B$ with balls $B_{i}=\lambda B\left(p_{i}\right)$ with $p_{i} \in B$ for $i=1, \ldots, N$ such that the balls $\frac{1}{2} B_{i}$ are disjoint and inside $B$. Let $\frac{1}{2} B_{1}=\frac{1}{2} \lambda B\left(p_{1}\right)$ the one of smallest volume among $\frac{1}{2} B_{1}, \ldots, \frac{1}{2} B_{N}$. Since $B \subset 2 B\left(p_{1}\right)$, we have

$$
\operatorname{vol}\left(2 B\left(p_{1}\right)\right) \geq \operatorname{vol}(B) \geq \sum_{i=1}^{N} \operatorname{vol}\left(\frac{1}{2} B_{1}\right) \geq N \cdot \operatorname{vol}\left(\frac{1}{2} B_{1}\right)=N \cdot \operatorname{vol}\left(\frac{1}{2} \lambda B\left(p_{1}\right)\right) .
$$

On the other hand, by Bishop-Gromov (cf. Corollary 5.6),

$$
\left.\frac{\operatorname{vol}\left(2 B\left(p_{1}\right)\right)}{\operatorname{vol}\left(\frac{1}{2} \lambda B\left(p_{1}\right)\right.}\right) \leq \frac{\omega_{n} \cdot(2 r)^{n}}{\omega_{n} \cdot\left(\frac{1}{2} \lambda r\right)^{n}}=\left(\frac{4}{\lambda}\right)^{n}
$$

thus

$$
N \leq\left(\frac{4}{\lambda}\right)^{n}=\left(2 L \cdot 10^{n+2}\right)^{n} .
$$

By Step 3, all $B_{i}^{m}:=10^{m} B_{i}$ for $m=0, \ldots, n+1$ have corank $\geq k$ and thus $\operatorname{cont}\left(B_{i}\right) \leq a_{k}$. Since the radii of the $B_{i}=B_{i}^{0}$ are very small, we have (by triangle inequality)

$$
B \subset \bigcup_{i} B_{i} \subset \bigcup_{i} 10^{n+1} B_{i} \subset 5 B
$$

hence

$$
\operatorname{cont}(B) \leq \operatorname{cont}\left(\bigcup_{i} B_{i}, \bigcup_{i} 10^{n+1} B_{i}\right)
$$

We will see below (cf. Appendix) that we may estimate

$$
\operatorname{cont}\left(\bigcup_{i} B_{i}, \bigcup_{i} 10^{n+1} B_{i}\right) \leq \sum_{j=1}^{n+1} \sum_{i_{1}<\ldots<i_{j}} \operatorname{cont}\left(\bigcap_{p=1}^{j} B_{i_{p}}^{n-j+1}, \bigcap_{p=1}^{j} 10 B_{i_{p}}^{n-j+1}\right)
$$

where $B_{i}^{m}:=10^{m} B_{i}$. Since

$$
\bigcap_{p=1}^{j} B_{i_{p}}^{m} \subset B_{i_{1}}^{m} \subset 5 B_{i_{1}}^{m} \subset \bigcap_{p=1}^{j} 10 B_{i_{p}}^{m}
$$

we get

$$
\operatorname{cont}\left(\bigcap_{p=1}^{j} B_{i_{p}}^{m}, \bigcap_{p=1}^{j} 10 B_{i_{p}}^{m}\right) \leq \operatorname{cont}\left(B_{i_{1}}^{m}\right) \leq a_{k}
$$


There are $N$ balls $B_{1}^{m}, \ldots, B_{N}^{m}$, so there are at most $N^{n}$ intersections $B_{i_{1}}^{m} \cap \ldots \cap B_{i_{j}}^{m}$ where $j=1, \ldots n$. Thus

$$
\operatorname{cont}(B) \leq N^{n+1} a_{k}
$$

which finishes the proof by (8.19)

Step 5. Let $a_{k}$ still denote an upper bound for the content of any ball with corank $\geq k$. By Step 1 we may choose $a_{K}=1$ where $K \leq c:=c(L, n)$ is the biggest possible corank. Thus by Step $4, a_{K-1}=\chi$, hence $a_{K-2}=\chi^{2}$ and eventually (by induction), $a_{0}=\chi^{K}$. Hence we get for any ball $B$

$$
\operatorname{cont}(B) \leq \chi^{c}
$$

which finishes the proof since $b(M)=\operatorname{cont}(B)$ for some big ball (cf. (8.16)).

Remark 8.12 The highest known total Betti number for a manifold with $K \geq 0$ is $2^{n}$, the total Betti number of the n-dimensional torus $T^{n}=S^{1} \times \ldots \times S^{1}$. 


\section{Convexity.}

Definition 9.1 Let $M$ be a Riemannian manifold. A continuous function $f: M \rightarrow \mathbb{R}$ is called convex if $f \circ \gamma: I \rightarrow \mathbb{R}$ is convex for any geodesic $\gamma: I \rightarrow M$, and $f$ is called concave if $-f$ is convex.

Theorem 9.2 A continuous function $f: M \rightarrow \mathbb{R}$ is convex if for any $x \in M$ and $\varepsilon>0$ there is a smooth lower support function $f_{x, \varepsilon}=\tilde{f}: U_{x} \rightarrow \mathbb{R}$ (i.e., $\tilde{f} \leq f, \tilde{f}(x)=f(x)$ ), defined in a neighborhood $U_{x} \subset M$, with $D \nabla \tilde{f}(x)>-\varepsilon$.

Proof. Suppose there is a geodesic $\gamma: I \rightarrow M$ such that $g=f \circ \gamma$ is not convex. Then there exists a parabola $a: \mathbb{R} \rightarrow \mathbb{R}$ with:

1) $a^{\prime \prime} \equiv-\varepsilon$

2) $a\left(t_{1}\right)=g\left(t_{1}\right)$ and $a\left(t_{2}\right)=g\left(t_{2}\right)$

3) there exists $t \in\left(t_{1}, t_{2}\right)$ with $a(t)<g(t)$.

Fig. 12.

Define $\delta=g-a$; then $\delta$ takes a maximum at some point $t_{0} \in\left(t_{1}, t_{2}\right)$, and we have $\delta\left(t_{0}\right)>0$. Consider $\tilde{f}=f_{x, \varepsilon}$ with $x=\gamma\left(t_{0}\right)$ and $\varepsilon$ small enough. Then $\tilde{g}=\tilde{f} \circ \gamma$ is a lower support function for $g$ at $t_{0}$, hence $(\tilde{g}-a)$ also takes a maximum at $t_{0}$. But $g^{\prime \prime}\left(t_{0}\right)>-\varepsilon$ and $a^{\prime \prime}\left(t_{0}\right)=-\varepsilon$, hence

$$
(\tilde{g}-a)^{\prime \prime}\left(t_{0}\right)>-\varepsilon+\varepsilon=0
$$

which is impossible at a maximum point.

Remark 9.3 By the theorem, a $C^{2}$-function $f$ is convex if $D \nabla f \geq 0$. If $f$ is only continuous but satisfies the assumptions of Theorem 9.2 , we say $D \nabla f \geq 0$ in the sense of support functions. 
Example 9.4 Fix $o \in M$ and let $\rho(x)=|o, x|$. Then $D \nabla \rho=A(\rho)>0$ for small $\rho>0$ by example 2.3. However, $\rho$ is not smooth at $o$, but $\rho^{2}$ is smooth $\left(\right.$ since $\rho\left(\exp _{o}(v)\right)^{2}=\langle v, v\rangle$ if $\|v\|$ is small, cf. (6.1)), and it satisfies

$$
D \nabla\left(\rho^{2}\right)=2(d \rho \cdot \nabla \rho+\rho \cdot D \nabla \rho)>0,
$$

hence $\rho^{2}$ is convex near $o$.

Definition 9.5 A closed subset $C \subset M$ is called convex if any geodesic segment $\gamma$ in $M$ with end points on $C$ lies entirely in $C$. Clearly, if $f: M \rightarrow \mathbb{R}$ is a convex function, then the sublevel sets $M^{a}=\{x \in M \mid f(x) \leq a\}$ are convex subsets for all $a \in \mathbb{R}$. Note that the notion of convexity depends on the surrounding manifold. For example, in the cylinder $S^{1} \times \mathbb{R}$ with radius 1 , a metric ball of radius $r<\pi$ is not convex, but it is convex in a slightly larger ball of radius $r+\varepsilon<\pi$ inside the cylinder.

Definition 9.6 A smooth hypersurface $S=\partial B \subset M$ is called convex hypersurface if for the interior unit normal vector field $N$,

$$
D N \leq 0
$$

Clearly, if $f$ is smooth and convex, then $S=\partial M^{a}$ is a convex hypersurface unless $a$ is a minimum of $f$ (note that $\nabla f$ can vanish only at a minimum of a convex function $f$ ). Vice versa, if $S=\partial B$ is a strictly convex hypersurface, i.e. $D N<0$, then the signed distance function $\rho=\rho_{S}= \pm|, S|$ is concave near $S$ since $D N$ is its Hessian along $S$ (cf. Ch.2, p.9). Consequently, $C=C \operatorname{los}(B)$ is convex in a neighborhood of $C$ since it is a sublevel set of the convex function $-\rho$.

Theorem 9.7 Let $B$ be compact and $S=\partial B$ a convex hypersurface. If $K \geq 0$ on $B$, then $\rho=|, S|$ is concave on all of $B$.

Proof. Let $x \in B$ and $\gamma$ a shortest geodesic from $x$ to $S$. Let $p \in S$ be its endpoint. If we were in euclidean space, there would be a support hyperplane for $B$ at $p$. In $M$ we have a similar construction: For large $R$, let $\tilde{S}=\exp _{p}\left(\partial B_{R}\left(-R N_{p}\right) \cap U\right)$ where $U$ is a small neighborhood of $-R N_{p}$ in $T_{p} M$. We will show that $\tilde{S}$ supports $B$ at $p$, i.e. $\tilde{S} \cap B=\emptyset$ and $\tilde{S} \cap \partial B=\{p\}$. To this end, we compare the signed distance functions $\rho$ of $S$ and $\tilde{\rho}$ of $\tilde{S}$ near $p$. Since $N_{p}$ is a common normal vector for $S$ and $\tilde{S}$, these functions agree at $p$ up to first derivatives. The second derivatives (Hessian) of $\rho$ and $\tilde{\rho}$ are given by the shape operators $A=(D N)_{p}$ and $\tilde{A}=(D \tilde{N})_{p}$ of $S$ and $\tilde{S}$. By convexity, we have $A \leq 0$. On the other hand, $\tilde{A}=\frac{1}{R} \cdot I>0$. (In fact, this holds for the euclidean sphere $\partial B_{R}\left(-R N_{p}\right) \subset T_{p} M$ and hence also for $\tilde{S}$ since $\exp _{p}$ preserves the covariant derivative 
$D$ at $p$, cf. Remark 1.5.) Thus $A<\tilde{A}$. However, the Hessians of $\rho$ and $\tilde{\rho}$ are both zero in $N_{p}$-direction, so we have no strict inequality. This can be repaired by passing to functions $f \circ \rho$ and $g \circ \tilde{\rho}$ which have the same level hypersurfaces as $\rho$ and $\tilde{\rho}$, where we choose the functions $f, g: \mathbb{R} \rightarrow \mathbb{R}$ monotone with

$$
f(0)=g(0), f^{\prime}(0)=g^{\prime}(0), f^{\prime \prime}(0)<g^{\prime \prime}(0) .
$$

Then $f \circ \rho$ and $g \circ \tilde{\rho}$ agree at $p$ up to first derivatives with $\operatorname{Dd}(f \circ \rho)_{p}<\operatorname{Dd}(g \circ \tilde{\rho})_{p}$, so we get $f \circ \rho \leq g \circ \tilde{\rho}$ near $p$ (in fact, " $<$ " outside $p$ ). In particular, the level set $\tilde{S}=\{g \circ \tilde{\rho}=0\}$ lies outside $B=\{f \circ \rho>0\}$.

Fig. 13.

Now we consider the signed distance function $\tilde{\rho}$ of $\tilde{S}$ on its full domain, namely wherever the mapping

$$
S \times \mathbb{R} \rightarrow M:(s, t) \mapsto \exp (t N(s))
$$

is invertible, cf. Ch.2, p.8. In particular, $\rho$ is defined and smooth near $x$ : Since $\gamma$ is a shortest curve from $p$ to $S$, there are no focal points for $\mathrm{S}$ on $\gamma$ between $p$ and $x$. Since $\tilde{A}>A$ at $p$, the focal distance for $\tilde{S}$ along $\gamma$ is strictly larger than for $S$ (cf. Remark 3.2 ), hence $x$ is no focal point for $\tilde{S}$, and $\tilde{\rho}$ is defined and smooth near $x$.

Since $\tilde{S}$ lies outside $B$, any curve from $B$ to $\tilde{S}$ meets $S=\partial B$ first, so $\tilde{\rho}$ is an upper support function for $\rho$ at $x$. Moreover, by the comparison theorem 3.1 we have

$$
D \nabla \tilde{\rho}(x) \leq \frac{1}{R+\tilde{\rho}(x)}=: \varepsilon
$$

so $-\rho_{S}$ is convex on $B$ by theorem 9.1 . 
Remark 9.8 The proof shows that $S=\partial B$ need not to be smooth; it is sufficient that there is a unit vector $N_{p} \in T_{p} M$ such that $\tilde{S}_{p, R}:=\exp _{p}\left(\partial B_{R}\left(-R N_{p}\right) \cap U\right)$ supports $B$ for any $p \in S, R>0$ and a neighborhood $U$ of $-R N_{p}$ in $T_{p} M$. For example, this is true if $C \operatorname{los}(B)=: C$ is convex (with $B=\operatorname{Int}(C)$ ). To see this, let $p \in \partial C$ and consider the tangent cone

$$
T_{p} C=C l o s\left\{v \in T_{p} M ; \exp _{p}(t v) \in C \text { for small } t>0\right\} .
$$

By convexity of $C$, this is a convex cone in $T_{p} M$ (since in exponential coordinates, $D$ geodesics and $\partial$-geodesics, i.e. straight lines, are close to each other near the origin, see 1.5), hence it is contained in some closed half space $H_{N}=\left\{v \in T_{p} M ;\langle v, N\rangle \geq 0\right\}$. Any such vector $N=N_{p}$ is called an inner normal vector of $\partial C$ at $p$, and $-N_{p}$ is called an outer normal vector. By strict convexity of $\tilde{S}=\tilde{S}_{p, R}$, the set $\tilde{D}=\exp _{p}\left(D_{R}\left(-R N_{p}\right) \cap U\right)$ is convex in a neighborhood of $p$, being a sublevel set of the convex function $-\tilde{\rho}$. Thus, $\tilde{D} \cap C$ is convex near $p$. But this shows that $\tilde{D} \cap C=\{p\}$, proving that $\tilde{S}$ supports $C$ at $p$. Namely, if $q=\exp _{p} v \in \tilde{D} \cap C$, then $\exp _{p} t v \in \tilde{D} \cap C$ for all $t \in[0,1]$, hence $v \in T_{p} C \cap D_{R}\left(-R N_{p}\right)=\{0\}$, so $q=p$. 


\section{Open manifolds with nonnegative curvature}

Theorem 10.1 (Cheeger - Gromoll 1970 [6])

Let $M$ an open (i.e. complete, noncompact) manifold with $K \geq 0$. There exists a compact convex submanifold (without boundary) $\Sigma \subset M$ called soul such that $M$ is diffeomorphic to the normal bundle $\nu \Sigma$.

Recall that the normal bundle of a submanifold $\Sigma \subset M$ is $\nu \Sigma=\cup_{p} \nu_{p} \Sigma$, where $\nu_{p} \Sigma=\left\{v \in T_{p} M \mid v \perp T_{p} \Sigma\right\}$. The proof needs some preparations. For the moment, assume only that $M$ is complete and noncompact.

Definition 10.2 A ray is a geodesic $\gamma$ defined on $[0, \infty)$ which is a shortest geodesic between any two of its points.

Remark 10.3 For any $p \in M$ there exists a ray starting at $p$ : Consider a sequence of points $q_{i}$ such that $\left|q_{i}, p\right| \rightarrow \infty$. Consider shortest geodesics $\gamma_{i}$ from $p$ to $q_{i}$. There exists a subsequence of the unit tangent vectors converging to some $v \in S_{p} M$. The geodesic $\gamma_{v}$ in the direction of $v$ is a ray. Moreover, if the points $q_{i}$ lie on some geodesic ray $\gamma$, i.e. $q_{i}=\gamma\left(t_{i}\right)$ with $t_{i} \rightarrow \infty$, the geodesic ray $\gamma_{v}$ is called an asymptote of $\gamma$. Note that asymptotes are not necessarily unique.

Definition 10.4 The Busemann function associated to a ray $\gamma$ is defined as

$$
b_{\gamma}(x)=\lim _{t \rightarrow \infty}(|x, \gamma(t)|-t)
$$

In particular, $b_{\gamma}(\gamma(s))=\lim (|\gamma(s), \gamma(t)|-t)=\lim (t-s-t)=-s$. Further, since $\rho_{\gamma(t)}=|\cdot, \gamma(t)|$ are Lipschitz-continuous functions with Lipschitz constant $L=1$, the same holds for $b_{\gamma}(x)$. Its level sets are called horospheres.

Consider an asymptote $\gamma_{x}$ of the ray $\gamma$. We define

$$
b_{x, t}(y)=\left|\gamma_{x}(t), y\right|-t+b_{\gamma}(x) .
$$

$b_{x, t}$ is smooth in a neighborhood of $p$ since $x$ is not in the cut locus of any point on $\gamma_{x}$.

Lemma $\mathbf{1 0 . 5} b_{x, t}$ is a support function of $b_{\gamma}$ at $x$.

Proof. There is a sequence $t_{i} \rightarrow \infty$ such that $\gamma_{x}=\lim \gamma_{i}$ where $\gamma_{i}$ is a shortest geodesic from $x$ to $\gamma\left(t_{i}\right)$. Then by triangle inequality, we have for any $y \in M$ :

$$
\begin{aligned}
b_{x, t}(y) & =\left|\gamma_{x}(t), y\right|-t+b_{\gamma}(x) \\
& \approx\left|\gamma_{i}(t), y\right|-t+b_{\gamma}(x) \\
& \geq\left|\gamma\left(t_{i}\right), y\right|-s_{i}+b_{\gamma}(x)
\end{aligned}
$$


Fig. 14 .

where $s_{i}=\left|x, \gamma\left(t_{i}\right)\right|$. The sign " $\approx "$ means that the error can be made as small as one wants $($ as $i \rightarrow \infty)$. From

$$
b_{\gamma}(x) \approx\left|x, \gamma\left(t_{i}\right)\right|-t_{i}=s_{i}-t_{i}
$$

we get

$$
\begin{aligned}
b_{x, t}(y) & \geq\left|y, \gamma\left(t_{i}\right)\right|-t_{i} \\
& \approx b_{\gamma}(y) .
\end{aligned}
$$

Moreover, $b_{x, t}(x)=b_{\gamma}(x)$. So $b_{x, t}$ is a support function to $b_{\gamma}$.

Lemma 10.6 If $M$ is complete, noncompact with $K \geq 0$, then each Busemann function $b_{\gamma}(x)$ is concave.

Proof. It follows from the comparison theorem 3.1 with $k=0$ that

$$
D \nabla b_{x, t}(x) \leq \frac{1}{\left|x, \gamma_{x}(t)\right|} \rightarrow 0
$$

as $t \rightarrow \infty$. So by Theorem $9.2, b_{\gamma}$ is concave.

Corollary 10.7 The superlevel sets

$$
C_{t, \gamma}=\left\{x \in M \mid b_{\gamma}(x) \geq t\right\}
$$

are convex sets in $M$ for any $t \in \mathbb{R}$. 
For any point $p \in M$ we consider

$$
R_{p}=\{\text { rays } \gamma:[0, \infty) \rightarrow M, \quad \gamma(0)=p\}
$$

Define the function

$$
b=\min _{\gamma \in R_{p}} b_{\gamma} .
$$

(The infimum is a minimum since a limit of rays is a ray.) $b$ is concave since it is the minimum of concave functions, and therefore, its superlevel sets

$$
C_{t}=\{x \in M \mid b(x) \geq t\} .
$$

are convex.

Lemma $10.8 C_{t}$ is compact.

Proof. Assume first $t \leq 0$. If $C_{t}$ were not compact, there whould exist a sequence of points $q_{i} \rightarrow \infty$ in $C_{t}$. Since $b(p)=0$, we have $p \in C_{t}$. For any $i$, choose a shortest geodesic segment $\gamma_{i}$ from $p$ to $q_{i}$; since $C_{t}$ is convex, $\gamma_{i}$ is contained in $C_{t}$. Since $C_{t}$ is closed, the limit ray $\gamma=\lim _{i} \gamma_{i}$ again lies in $C_{t}$. But $\gamma$ is a ray starting at $p$, so $\gamma \in R_{p}$. But then $\gamma$ cannot be contained in $C_{t}$ since $b(\gamma(s)) \leq b_{\gamma}(\gamma(s))=-s$ can be made smaller than $t$, contradiction!

Since $C_{s} \subset C_{t}$ for $s>t$, all superlevel sets of $b(x)$ are compact.

Now let $t_{0}$ be the maximum value of $b$ (which exists by compactness). Define $C^{1}=C_{t_{0}} . C^{1}$ cannot contain interior points. (In fact, if $x \in C^{1}$ and $b(x)=b_{\gamma}(x)$, then $b_{\gamma}$ decreases with speed one along an asymptotic ray $\gamma_{x}$ of $\gamma$. Thus $b \leq b_{\gamma}$ cannot stay maximal near $x$.) Thus $C^{1}$ is a compact convex set of lower dimension. In general, a compact convex subset $C$ of a Riemannian manifold is a subset of a (non complete) submanifold $M^{1}$, such that $C$ has nonempty interior relative to $M^{1}$ (cf. [5]). If $\partial C^{1} \neq \emptyset$, we consider the distance function $\rho_{\partial C_{1}}$ on $C^{1}$. Since $C^{1}$ is convex, $\partial C^{1}$ is a convex hypersurface. ¿From Theorem 9.7, and Remark 9.8 we see that $\rho_{\partial C_{1}}$ is concave on $C^{1}$, thus its superlevel sets

$$
C_{t}^{1}=\left\{\rho_{\partial C_{1}} \geq t\right\}
$$

are convex again. If $t_{1}$ is the maximum of $\rho_{\partial C_{1}}$, the set

$$
C^{2}=C_{t_{1}}^{1}
$$

cannot have interior points in $M^{1}$, thus is again of lower dimension. In this way we produce a descending chain

$$
C^{1} \supset C^{2} \supset \ldots \supset C^{k}
$$


of compact convex subsets with lower and lower dimension. This process ends after a finite number (say: $k$ ) of steps; if we put $\Sigma=C^{k}$, then $\Sigma$ is a compact convex set without boundary: the soul of $M$.

It remains to show that $M$ is diffeomorphic to $\nu \Sigma$. In fact, we will show that $M$ is diffeomorphic to a tubular neighborhood of $\Sigma$, say $B_{r}(\Sigma)$, for small $r$ which itself is diffeomorphic to $\nu \Sigma$ via the exponential map $\exp \mid \nu \Sigma$.

Lemma 10.9 For small $r>0$, there is a diffeomorphism $\varphi: M \rightarrow B_{2 r}(\Sigma)$ with $\varphi=i d$ on $B_{r}(\Sigma)$.

Proof. Let $\rho_{\Sigma}=|, \Sigma|$. We show first that $\rho_{\Sigma}$ has no critical points on $M \backslash \Sigma$, i.e. for any $x \in M \backslash \Sigma$ there is a gradientlike vector $v \in T_{x} M$ for $\rho_{\Sigma}$.

In fact, since $x \notin \Sigma$, there is $j \in\{1, \ldots, k-1\}$ such that $x \in C^{j} \backslash C^{j+1}$. In particular, there is some $t$ such that $x \in \partial C_{t}^{j}$. Now, since $\Sigma \subset C_{t}^{j}$, any geodesic $\gamma$ from $x$ to $\Sigma$ has initial vector $\gamma^{\prime}(0)$ pointing to the interior of $C_{t}^{j}$. Thus, an outer normal vector for the convex set $C_{t}^{j}$ is gradientlike.

Now for any $x \in M \backslash B_{r}(\Sigma)$, we choose a gradientlike vector $v \in N_{x} C_{t}$ and enlarge it to a gradientlike vector field $V_{x}$ on some neighborhood $U_{x}$. By paracompactness, we may pass to a locally finite subcovering $\left(U_{x_{i}}\right)_{i=1,2, \ldots}$. Let $V_{i}=V_{x_{i}}$. On $B_{2 r}(\Sigma) \backslash \Sigma$, we put $V_{0}=\nabla \rho_{\Sigma}$ : We have chosen $r>0$ so small that $\rho_{\Sigma}$ is smooth on $B_{2 r}(\Sigma)$; this is possible by compactness of $\Sigma$. We may choose a subordinated partition of unity $\left(\varphi_{i}\right)_{i \geq 0}$ : then $V=\sum \varphi_{i} V_{i}$ is a smooth gradientlike vector field defined on $M \backslash \Sigma$ which agrees to $\nabla \rho_{\Sigma}$ on $B_{r}(\Sigma) \backslash \Sigma$.

Let $c_{x}$ be the integral curve of $V$ with $c_{x}(0)=x$. Since the integral curves intersect $\partial B_{r}(\Sigma)$ transversally (in fact, orthogonally), there is a smooth function

$$
t: M \backslash \Sigma \rightarrow \mathbb{R}
$$

with

$$
c_{x}(t(x)) \in \partial B_{r}(\Sigma) .
$$

We reparametrize the integral curves and put

$$
\tilde{c}_{x}(t)=c_{x}(r+t(x)-t) .
$$

Now let $\chi: \mathbb{R}_{+} \rightarrow[0,2 r)$ smooth, with $\chi(t)=t$ for $t \in[0, r]$ and $\chi^{\prime}>0$, and let $\varphi: M \rightarrow B_{2 r}(\Sigma)$ with

$$
\varphi(x)= \begin{cases}x & x \in B_{r}(\Sigma) \\ \tilde{c}_{x}(\chi(r+t(x))) & \text { otherwise }\end{cases}
$$

Then $\varphi$ maps diffeomorphically $M$ onto $B_{2 r}(\Sigma)$. 
Fig. 15.

Theorem 10.10 (Perelman) Let $M$ be an open manifold of $K \geq 0$ with soul $\Sigma$. For any $p \in \Sigma$ and any two nonzero vectors $a \in T_{p} \Sigma, v \in \nu_{p} \Sigma$, there is a totally geodesic flat half plane through $p$ tangent to $a$ and $v$.

The proof uses the contraction of Sharafutdinov ([32],[35]) which is a continuous mapping $\phi: M \rightarrow \Sigma$ with

$$
|\phi(x), \phi(y)| \leq|x, y|
$$

for all $x, y \in M$. In fact, $\phi$ is the limit of an iterated projection onto more and more smaller and smaller convex sets surrounding $\Sigma$, cf. [35] for details.

Proof of Perelman's theorem: Let

$$
F=\phi \circ \exp : \nu \Sigma \rightarrow \Sigma
$$

and put

$$
f(v)=|\pi(v), F v|
$$

for $v \in \nu \Sigma$, where $\pi: \nu \Sigma \rightarrow \Sigma$ denotes the projection. Consider

$$
m f(t):=\max \left\{f(v) ; \quad v \in \nu^{t} \Sigma\right\}
$$

where $\nu^{t} \Sigma=\{v \in \nu \Sigma ;\|v\|=t\}$. Clearly $m f(0)=0$ and $m f(t) \geq 0$ for all $t \geq 0$. We claim that $m f(t)$ is monotonely decreasing so that we get $m f \equiv 0$. Let $t>0$ be small enough such that $m f(t)$ is strictly less than the cut locus distance on $\Sigma$. Let $v \in \nu^{t} \Sigma$ so that $f(v)=m f(t)$. Let $\alpha$ be the shortest geodesic in $\Sigma$ from $F(v)$ to $p=\pi(v)$. By assumption, the prolongation of $\alpha$ stays shortest beyond $p$ up to some point $q \in \Sigma$. Let $w \in \nu_{q} \Sigma$ be the parallel displacement of $v$ from $p$ to $q$ along $\alpha$. 
Fig. 16.

Lemma 10.11 We have $f(w)=m f(t)$ and $|F v, F w|=|p, q|$, and $p, q$, exp $v$, exp $w$ span a totally geodesic flat rectangle $R$.

Proof. By the contraction property of $\phi$ and Rauch II we have

$$
|F v, F w| \leq|\exp v, \exp w| \leq|p, q|
$$

and moreover, by the maximality of $f(v)=m f(t)$,

$$
|F w, q|=f(w) \leq m f(t) .
$$

On the other hand, $F v, p$ and $q$ are lined up on a shortest geodesic, so

$$
|F v, q|=m f(t)+|p, q| .
$$

So the triangle inequality for $F v, F w, q$ yields equality in (10.9) and (10.10) and we have proved the Lemma, using the equality case of Rauch II (cf. Corollary 3.4).

Now we consider the vector $w^{\prime}=(1-(\varepsilon / t)) w$ with length $\left\|w^{\prime}\right\|=\|w\|-\varepsilon=t-\varepsilon$. Clearly

$$
m f(t-\varepsilon) \geq f\left(w^{\prime}\right)=\left|q, F w^{\prime}\right| .
$$

Since $R=(p, \exp v, \exp w, q)$ is a flat rectangle,

$$
\left|\exp v, \exp w^{\prime}\right|^{2} \leq|\exp v, \exp w|^{2}+\varepsilon^{2}=|p, q|^{2}\left(1+\left(\frac{\varepsilon}{|p, q|}\right)^{2}\right),
$$

hence

$$
\left|F v, F w^{\prime}\right| \leq\left|\exp v, \exp w^{\prime}\right| \leq|p, q|+C \varepsilon^{2}
$$


Fig. 17.

with $C=(2|p, q|)^{-1}$. Using (10.10),(10.11) and the triangle inequality for $F v, q, F w^{\prime}$ we get

$$
m f(t-\varepsilon) \geq\left|q, F w^{\prime}\right| \geq|F v, q|-\left|F v, F w^{\prime}\right| \geq m f(t)-C \varepsilon^{2} .
$$

This implies that $m f$ is monotonely decreasing and hence zero. Thus $f \equiv 0$ and $\phi(\exp v)=\pi(v)$ for any $v \in \nu \Sigma$. (Note that the condition for $t=\|v\|$ made at the beginning now is void.) In particular, $|\exp v, \exp w|=|\pi v, \pi w|$ for any two normal vectors $v, w$ on $\Sigma$ which are parallel along a shortest geodesic on $\Sigma$. So the proof is finished by the equality case of Rauch II (Corollary 3.4).

Remark 10.12 The above theorem also proves a conjecture of Cheeger and Gromoll saying that the soul must be a point (and hence $M$ must be diffeomorphic to $\mathbb{R}^{n}$ ) provided that there is a point $q \in M$ where all sectional curvatures are strictly positive. In fact, we can connect $q$ to $\Sigma$ by a shortest geodesic $\gamma$ (which is perpendicular to $\Sigma$ ). If $\Sigma$ has positive dimension, i.e. if there is a nonzero tangent vector $a$ of the soul where it meets $\gamma$, then by Perelman's theorem, there is a flat totally geodesic half plane spanned by $a$ and $\gamma$. Thus not all curvatures at $q=\gamma(0)$ are positive. - We are indepted to V. Schroeder for communicating Perelman's proof.

Remark 10.13 By a previous result of Strake and Walshap [33], Perelman's theorem implies that on a small tubular neighborhood of the soul, the projection $\pi: B_{r}(\Sigma) \rightarrow \Sigma$ is a Riemannian submersion, i.e. $d \pi_{x}$ is an orthogonal projection up to isometric linear isomorphisms, for any $x \in B_{r}(\Sigma)$. We conjecture that the Sharafutdinov contraction $\phi: M \rightarrow \Sigma$ is smooth and also a Riemannian submersion. 


\section{The sphere theorem.}

One of the most celebrated results in Riemannian geometry is the "sphere theorem"; cf. [2], [5], [11], [18], [25], [28].

Theorem 11.1 (Rauch, Berger, Klingenberg)

Let $M^{n}$ be a compact, simply connected manifold, with $K>0$. Assume that

$$
\frac{\max K}{\min K}<4
$$

Then $M$ is homeomorphic to $S^{n}$.

Remark 11.2 The estimate 4 is sharp. There are simply connected manifolds with

$$
\frac{\max K}{\min K}=4
$$

which are not homeomorphic to $S^{n}$, namely the projective spaces over the fields $\mathbb{C}$ and $\mathbb{H}$ (called $\mathbb{C} P^{m}$ and $\mathbb{H} P^{m}$ ), and the Cayley projective plane.

Remark 11.3 Another type of sphere theorem using a diameter estimate instead of the upper curvature bound was given by Grove and Shiohama ([21], [28]).

Proof. We may assume (rescaling the metric) that

$$
\frac{1}{4}<K<1
$$

so the comparison spaces are the spheres $S_{2}$ and $S_{1}$ with radii 2 and 1 respectively. We fix $p \in M$, and consider geodesics of length $\pi$ on the three manifolds.

Fig. 18. 
By the upper curvature bound, the first conjugate point comes later than on $S_{1}$, hence the exponential map $\exp _{p} \mid \overline{B_{\pi}(0)}$ is an immersion (local diffeomorphism).

By the lower curvature bound, the immersed hypersurface $f:=\exp _{p} \mid \partial B_{\pi}(0)$ is strictly concave, i.e. $D N<0$ for the exterior unit normal field $N$ (cf. Theorem 3.1)

Now we need Theorem 11.4 (see below) to finish the proof. Take two copies $D_{+}$, $D_{-}$of the unit disk $D^{n}$ and identify $D_{+}$with $D_{\pi}(0)=\overline{B_{\pi}(0)}$ and put $S=\partial B_{\pi}(0)$. By Theorem 11.4, there exists an immersion $F: D_{-} \rightarrow M$ and a local diffeomorphism $\varphi: S=\partial D_{+} \rightarrow \partial D_{-}$such that $f=F \circ \varphi$. Let

$$
\Sigma_{\varphi}=D_{+} \cup_{\varphi} D_{-}=\left(D_{+} \amalg D_{-}\right) / \sim,
$$

where the equivalence relation is given as follows: $x \in \partial D_{+}$and $y \in \partial D_{-}$are equivalent $(x \sim y)$ iff $y=\varphi(x)$.

Fig. 19.

This is a smooth manifold which is homeomorphic to $S^{n}$ (see figure). It is diffeomorphic if and only if $\varphi$ extends to a diffeomorphism $\phi: D_{+} \rightarrow D_{-}$. Now we define $\hat{F}: \Sigma_{\varphi} \rightarrow M$ by putting

$$
\hat{F}\left|D_{+}=\exp _{p}, \quad \hat{F}\right| D_{-}=F
$$

Then $\hat{F}$ is a local diffeomorphism. It is onto because the image is open and closed and it is injective, since $M$ is simply connected. Hence $\hat{F}$ is a diffeomorphism.

Remark 11.4 In dimension $n=2$ we cannot apply the above proof. However, the theorem follows from the Gauss-Bonnet formula: Since $K>0$, it follows that

$$
0<\int_{M} K=2 \pi \chi(M)
$$


thus $\chi(M)=(2-2 g)>0$, which implies that $g=0$ where $g$ is the genus of $M$. Hence $M$ is a sphere.

Theorem 11.5 (Gromov, Eschenburg [11])

Let $M^{n}, n \geq 3$, be a complete manifold with $K \geq 0$. Let $S$ be a compact $(n-1)$ dimensional manifold, and $f: S \rightarrow M$ an $\varepsilon$-convex immersion, i.e. we suppose that a unit normal vector field $N$ along $f$ satisfies $D N<-\varepsilon$. Then $f$ bounds an immersed convex disk, i.e. there exists an immersion

$$
F: D^{n} \rightarrow M
$$

(where $D^{n}$ is the unit $n$-disk) and a diffeomophism

$$
\varphi: S \rightarrow \partial D^{n}
$$

such that $f=\varphi \circ F$ and $N \circ \varphi^{-1}$ becomes the interior normal field.

If $M=\mathbb{R}^{n}$, we get the following stronger theorem which is due to Hadamard [22]:

Theorem 11.6 (Hadamard)

Let $S$ be a compact $(n-1)$-manifold, and $f: S \rightarrow \mathbb{R}^{n}$ an $\varepsilon$-convex immersion. Then $f$ is an embedding and $f(S)$ bounds a convex $n$-disk.

Proof. Let $N$ be the unit normal field, considered as Gauss map $N: S \rightarrow S^{n-1}$. Since $S$ is strictly convex, $d N$ has only positive eigenvalues (note that $D N=d N$ where $D$ is the Levi-Civita derivative on $\mathbb{R}^{n}$, i.e the ordinary derivative $D=\partial$ ), so $N$ is a local diffeomorphism. But $S^{n-1}$ is simply connected for $n \geq 3$, hence $N$ is a diffeomorphism. So any vector $v \in S^{n-1}$ arises exactly twice as a normal vector for $f$, namely $v=N(x)=-N(y)$ for exactly two points $x, y \in S$. Thus, any height function $\langle f, v\rangle$ has exactly two critical points: one maximum and one minimum. From this we see that $f$ is injective: Since the height function $\langle f, v\rangle$ for $v=N(x)$ attains its maximum at $x$, there is no other point $y \in S$ with $\langle f(y), v\rangle=\langle f(x), v\rangle$ (otherwise there would be a second maximum). Moreover, we see that $f(S)$ bounds the convex set

$$
C=\bigcap_{x \in S} H(x)
$$

where $H(x)$ is the half space

$$
H(x)=f(x)+\left\{v \in \mathbb{R}^{n} ;\langle v, N(x)\rangle \leq 0\right\} .
$$


Remark 11.7 If $M \neq \mathbb{R}^{n}$, it is possible to construct an $\varepsilon$-convex immersion that is not injective. For example, take the cylinder $S^{1} \times \mathbb{R}$ with radius 1 , and consider the immersion $\exp _{p} \mid \partial B_{r}(0)$ for $r>\pi$ (for arbitrary $p \in M$ ).

Fig. 20.

Remark 11.8 Theorems 11.4 and 11.5 are false for $n=2$ : a counterexample is given by any locally strongly convex closed curve with winding number $\geq 2$ in euclidean plane $M=\mathbb{R}^{2}$. However, Theorem 11.5 holds for $n=2$ (with the same proof) provided that the winding number of the curve is \pm 1 .

Remark 11.9 Theorem 11.4 is also false if the curvature can be negative. E.g. let $S$ be the boundary of a small tubular neighborhood around a closed geodesic in a manifold $M$ with $K<0$. This is $\varepsilon$-convex (by the comparison theorem 3.1), but diffeomorphic to $S^{1} \times S^{n-2}$, not to $S^{n-1}$.

Proof of Theorem 11.4. We are using the principle that in spaces with $K \geq 0$, we do not loose convexity by passing to interior parallel hypersurfaces (cf. Theorem 9.7).

Let $S^{\prime}$ be an embedded piece of $f(S)$. Then by 9.7, $S^{\prime}$ bounds (partially) some open set $B$ on which $\rho=\rho_{S^{\prime}}$ is concave. However, $\rho$ is not strictly concave since it grows linearly along geodesics which are normal to $S^{\prime}$. This can be improved similarly as in the proof of Toponogov's theorem (Ch.6): Instead of $\rho$ consider

$$
\sigma=\frac{1}{2}(R-\rho)^{2}
$$

where $R:=1 / \varepsilon$. We compare with $\tilde{S}^{\prime}=\partial B_{R}(0) \subset \mathbb{R}^{n}$; there we have

$$
\tilde{\sigma}(x)=\frac{1}{2}(R-\tilde{\rho}(x))^{2}=\frac{1}{2}<x, x>,
$$


hence $D \nabla \tilde{\sigma}=I$. Therefore we get from the comparison theory (cf. 3.1)

$$
D \nabla \sigma \geq I
$$

in the sense of support functions (cf. Ch.9). Using convolution, we make $\sigma$ smooth: Let

$$
\sigma_{\delta}(x)=\int_{T_{x} M} \sigma\left(\exp _{x}(u)\right) \varphi_{\delta}(\|u\|) d u
$$

where $\varphi_{\delta}$ is a mollifier with support in $B_{\delta}(0)$, i.e. $\varphi_{\delta}: \mathbb{R}_{+} \rightarrow \mathbb{R}_{+}$is smooth with $\varphi_{\delta}=$ const near 0 , further $\varphi_{\delta}=0$ on $[\delta, \infty)$ and $\int \varphi_{\delta}(\|x\|) d^{n} x=1$. Now $\sigma_{\delta}$ is a smooth function with

$$
\left|\sigma_{\delta}(x)-\sigma(x)\right| \leq \delta,\left\|\nabla \sigma_{\delta}\right\| \leq R, D \nabla \sigma_{\delta} \geq 1
$$

where an arbitrary small error (which goes to zero as $\delta \rightarrow 0$ ) is allowed in these estimates. (The second estimate comes from the Lipschitz constant of $\sigma$ which is the maximum of $\frac{d}{d \rho}\left(\frac{1}{2}(R-\rho)^{2}\right)$ for $\rho>0$.) Thus we have lost no convexity. In fact, we do not use $\sigma_{\delta}$ itself but a combination $\chi(\rho) \sigma+(1-\chi(\rho)) \sigma_{\delta}$ for a suitable function $\chi$; this agress to $\sigma$ close to $S^{\prime}$ (for small $\rho$, smaller than the focal radius) and it agrees to $\sigma_{\delta}$ if $\rho$ is big enough. This has the same good properties as $\sigma_{\delta}$ and additionally, it has $S^{\prime}$ as the level hypersurface with value $\frac{1}{2} R^{2}$. Let us keep the name $\sigma_{\delta}$ for this modified function.

If $S$ were embedded, we could thus contract $S$ (using the level sets of $\sigma_{\delta}$ ) to a small $\varepsilon$-convex hypersurface lying in a small neighborhood of the origin of some exponential coordinate chart. Since $D \approx \partial$ near the origin for exponential coordinates (cf. Ch.1), this hypersurface is still $\varepsilon$-convex with respect to the euclidean geometry of the coordinate chart (for some smaller $\varepsilon$ ). So it bounds a disk (by 11.5).

However, $\sigma$ and $\sigma_{\delta}$ are defined only in a small neighborhood of $S^{\prime}$. But we may cover $S$ with open subsets $S_{i}$ such that $S_{i}^{\prime}=f \mid S_{i}$ are embedded. Thus we recieve corresponding functions $\left(\sigma_{\delta}\right)_{i}$ defined near $S_{i}^{\prime}$ which we may past together along the immersed hypersurface $f(S)$ : Note that the constant $\delta$ can be chosen independent of $i$, by compactness of $S$, and therefore $\left(\sigma_{\delta}\right)_{i}$ and $\left(\sigma_{\delta}\right)_{j}$ agree near $f\left(S_{i} \cap S_{j}\right)$. Now we may pass to the level hypersurfaces $\left\{\left(\sigma_{\delta}\right)_{i}=\frac{1}{2}(R-a)^{2}\right\}$ for some $a>0$ independent of $i$ which can be pasted together to an immersion $f_{1}: S \rightarrow M$. This new immersion $f_{1}$ has roughly distance $a$ from $f$ and is still $\varepsilon$-convex; in fact it is $\varepsilon_{1}$-convex with $\varepsilon_{1}=1 / R_{1}$ and $R_{1} \approx R-a$. Moreover, the gradient lines of the local $\sigma_{\delta}$ 's define a diffeomorphism between the hypersurfaces $f$ and $f_{1}$ which is length decreasing by a factor $\leq e^{-a / R}$, due to the estimates (11.11). We will show that we can iterate the process with the same number $a$. Then, after $N$ steps, $R_{N} \approx R-N a$ becomes arbitrarily small, so our immersion $f_{N}$ gets arbitrarily small. So it is still $\varepsilon$-convex in some exponential coordinates and hence by Hadamard's theorem (Theorem 11.6), $f_{N}$ is an embedding bounding a convex disk. Note that the whole process takes place in the relatively compact subset $M^{\prime}=B_{R}(f(S))$. 
However, in dimension $n=2$, if we started with an $\varepsilon$-convex closed curve of winding number $\geq 2$, we would get cusps and could not finish the contraction. The problem is that the embedded pieces $S_{i}$ become smaller and smaller as we approach the cusp, and thus the values $a$ must be chosen smaller and smaller. But we can exclude this behaviour in dimension $n \geq 3$.

Fig. 21.

Lemma 11.10 Let $M^{n}, n \geq 3$, be a complete manifold with $K \geq 0$ and $M^{\prime} \subset M$ a relatively compact open subset. There exists a constant $r>0$ depending only on $\varepsilon$ and on the geometry of $M^{\prime}$ such that, for any compact $(n-1)$-dimensional manifold $S$, for any $\varepsilon$-convex immersion $f: S \rightarrow M^{\prime}$, and for any $s \in S$, the connected component containing $s$ of the set $f^{-1}\left(B_{r}(f(s))\right.$ is embedded.

Proof. Let $f: S \rightarrow M^{\prime}$ as above and fix $s \in S$. Using exponential coordinates around $f(s)$, we find some $\delta>0$ (depending on $\varepsilon$ and the geometry of $M^{\prime}$ ) such that $f \mid S^{\prime}$ is still (say) $(\varepsilon / 2)$-convex with respect to the euclidean metric on $B_{2 \delta}(f(s)$ ) (in exponential coordinates), where $S^{\prime}$ denotes the connected component of $s$ in the open subset $f^{-1}\left(B_{2 \delta}(f(s)) \subset S\right.$. Replacing $\varepsilon / 2$ by $\varepsilon$ again, we have to consider now an $\varepsilon$-convex immersion $f: S^{\prime} \rightarrow \mathbb{R}^{n}$ with $f(s)=0$ such that $f^{-1}\left(\overline{B_{\delta}(0)}\right) \subset S^{\prime}$ is compact. We may assume that the unit normal vector at $s$ is the n-th coordinate vector, $N(s)=e_{n}$. Now we intersect $f\left(S^{\prime}\right)$ by horizontal hyperplanes (parallel to the tangent plane at $f(s)=0$ ), i.e. we look at the height function $h=x_{n} \circ f: S^{\prime} \rightarrow \mathbb{R}$.

By $\varepsilon$-convexity, the only critical points of $x_{n} \circ f$ are local maxima or minima, and $s$ is a local maximum with $h(s)=0$. Consider the flow lines of $-\nabla h$ (with respect to the induced metric on $S$ ) starting at $s$. Either, they reach the boundary $\partial B_{\delta}(0)$, or they end at a local minimum. If no flow line reaches the boundary, they end all at the same minimum since $S^{\prime} \backslash\{s\}$ is connected (here we need the dimension restriction $n-1 \geq 2$ ). 
Fig. 22.

In this case $S^{\prime}$ is compact (without boundary), so $S^{\prime}=S$, and $f$ is an embedding by Hadamard's theorem (cf. 11.5), and we are done.

So assume that some flow line of $-\nabla h$ reaches $\partial B_{\delta}(0)$. At which height is this possible? Along any of the flow lines, the heigth $h$ decreases. So let $-r<0$ be the highest $h$-value where some flow line reaches $\partial B_{\delta}(0)$. Then no flow line starting at $s$ ends at a minimum of height $>-r$ : otherwise, by connectedness of $S^{\prime}$, all flow lines would end at this minimum (they cannot reach the boundary before height $-r$ ) which we have excluded. Consider the connected component $S^{\prime \prime}$ of $\{h>-r\}$ which passes through $s$. Then still by Hadamard's theorem, $f \mid S^{\prime \prime}$ is an embedding, since the intersections of $f\left(S^{\prime \prime}\right)$ with the hyperplanes $\left\{x_{n}=t\right\} \cong \mathbb{R}^{n-1}$ for $0>t>-r$ are closed $\varepsilon$-convex hypersurfaces in $\mathbb{R}^{n-1}$ (which have winding number \pm 1 if $n-1=2$ since they contract to a point as $t \rightarrow 0$ ). To conclude the proof, we only have to find a good estimate for $r$.

\section{Claim.}

$$
f\left(S^{\prime \prime}\right) \subset B_{\delta}(0) \cap \overline{B_{R}\left(-R e_{n}\right)}
$$

where $R=1 / \varepsilon$, and consequently,

$$
r \geq \bar{r}=\frac{1}{2} \delta^{2} \varepsilon
$$

Proof. To see (11.13) from (11.12), note that $f\left(S^{\prime \prime}\right)$ cannot reach $\partial B_{\delta}(0)$ before $\partial B_{R}\left(-R e_{n}\right)$. So let $-\bar{r}$ denote the height where $\partial B_{R}\left(-R e_{n}\right)$ meets $\partial B_{\delta}(0)$. Then

$$
\delta^{2}-\bar{r}^{2}=R^{2}-(R-\bar{r})^{2}=2 \bar{r} R-\bar{r}^{2},
$$

hence $\bar{r}=\delta^{2} /(2 R)=\frac{1}{2} \delta^{2} \varepsilon$. 
Fig. 23.

Now we show (11.12). Put $S=f\left(S^{\prime \prime}\right) \subset \mathbb{R}^{n}$. (There is no danger of confusion with the previous $S$.) Note that $\bar{S}$ is a manifold with boundary $\partial S=\bar{S} \cap\left\{x_{n}=-r\right\}$. Let $\rho$ be the signed distance function from $S$ (continued negatively at the other side of $S$ ), defined on the open set $V \subset \mathbb{R}^{n}$ containing all points $x$ where a shortest line segment from $x$ to $S$ exists, i.e. which are closer to $S$ than to $\partial S$.

Fig. 24

As above, we put $\sigma=\frac{1}{2}(R-\rho)^{2}$. Then we have (cf. 11.9)

$$
D \nabla \sigma \geq I
$$

in the sense of support functions. (This holds also on the other side of $S$ since we are now in euclidean space.) On the other hand, we consider also $\tilde{\sigma}=\frac{1}{2}(R-\tilde{\rho})^{2}$ where $\tilde{\rho}$ is the signed distance function from $\tilde{S}:=\partial B_{R}\left(-R e_{n}\right)$. Hence $\tilde{\sigma}(x)=\left\|x-R e_{n}\right\|^{2}$ and

$$
D \nabla \tilde{\sigma}=I .
$$


Therefore $D \nabla(\sigma-\tilde{\sigma}) \geq 0$ which shows that $\sigma-\tilde{\sigma}$ is a convex function. Since $\sigma=\tilde{\sigma}$ and $\nabla \sigma=\nabla \tilde{\sigma}$ along $\mathbb{R}_{+} \cdot e_{n}$, there are critical points (hence minima) for $\sigma-\tilde{\sigma}$ along $\mathbb{R}_{+} \cdot e_{n}$. Thus we may conclude $(\sigma-\tilde{\sigma})(x) \geq 0$ for all $x \in V$ which can be connected to $\mathbb{R}_{+} \cdot e_{n}$ by a straight line segment inside $V$. We must show that this holds in particular for all $x \in S$.

Let $S_{1} \subset S$ be the set of points $x \in S$ where the vertical ray $x+\mathbb{R}_{+} \cdot e_{n}$ meets $S$ a second time and let $S_{2}=S \backslash S_{1}$. All the points above $S_{1}$ are in $V$ since they are closer to $S_{1}$ than to $\left\{x_{n}=-r\right\}$. Moreover, all points above $S_{2}$ are in $V$ for the same reason.

Fig. 25.

Thus we may connect any point of $S$ to some point in $\mathbb{R}_{+} \cdot e_{n}$ within the shaded region (cf. fig. 26 below); we just have to avoid the cylinder of height $r$ above $\partial S$ if we start from $S_{2}$. This finishes the proof of the claim, of the lemma and of the theorem.

Fig. 26. 


\section{Lower Ricci curvature bounds and the Maximum Principle.}

In this chapter, we want to discuss the following two theorems:

Theorem 12.1 (Myers-Cheng [31], [8])

Let $M$ be complete with

$$
\operatorname{Ric}(M) \geq n-1=\operatorname{Ric}\left(S^{n}\right) .
$$

Then $\operatorname{diam}(M) \leq \pi$, and equality holds if and only if $M$ is isometric to $S^{n}$.

Theorem 12.2 (Cheeger-Gromoll Splitting Theorem, [7], [13])

Let $M$ be complete with

$$
\operatorname{Ric}(M) \geq 0 \text {. }
$$

Then there exists a line in $M$, i.e. a complete geodesic which is shortest on any finite segment, if and only if $M$ is isometric to a Riemannian product $M^{\prime} \times \mathbb{R}$ for some complete $(n-1)$-manifold $M^{\prime}$ with $\operatorname{Ric}\left(M^{\prime}\right) \geq 0$.

By definition, the Riemannian product $M=M_{1} \times M_{2}$ of two Riemannian manifolds is the cartesian product with the metric

$$
\left\|\left(v_{1}, v_{2}\right)\right\|^{2}=\left\|v_{1}\right\|^{2}+\left\|v_{2}\right\|^{2}
$$

for any tangent vector $\left(v_{1}, v_{2}\right)$ of $M_{1} \times M_{2}$.

Let us first discuss the inequality of Theorem 12.1. This follows from our average comparison theorem 4.1: We saw that the first conjugate point on a geodesic $\gamma$ on $M$, i.e. the first singularity $t_{1}$ for a Riccati solution $A$ along $\gamma$ with $A(t) \sim \frac{1}{t} I$ near $t=0$, comes not later than on the comparison space $S^{n}$, i.e. at a distance $\leq \pi$. Beyond the first conjugate point, no geodesic can be shortest (cf. Ch.5), hence there are no shortest geodesics with length $>\pi$ and therefore the diameter is $\leq \pi$.

Corollary 12.3 A complete Riemannian manifold $M$ with Ric $\geq n-1$ is compact and has finite fundamental group.

Proof. Since $\operatorname{diam}(M) \leq \pi, M$ is the image of the compact set $\overline{B_{\pi}(0)} \subset T_{p} M$ under the map $\exp _{p}$ (for any $p \in M$ ), hence it is compact. By the same reason, the universal cover $\hat{M}$ is compact. Since a covering map of compact spaces has only finitely many preimages (otherwise, the preimages would accumulate), the covering $\hat{M} \rightarrow M$ is finite, hence $\pi_{1}(M)$ is finite. 
Remark 12.4 Under the same hypothesis, we also have

$$
\operatorname{Vol}(M) \leq \operatorname{Vol}\left(S^{n}\right)
$$

with equality if and only if $M$ is isometric to $S^{n}$. This follows immediately from the Bishop-Gromov inequality and its equality discussion.

Now let us come to the equality part of Theorem 12.1 and 12.2. These are rigidity theorems: the assumptions are so strong that we get a characterization up to isometries. The main ingredience is the Hopf-Calabi Maximum Principle for subharmonic functions:

Definition 12.5 Let $M$ be any Riemannian manifold and $f: M \rightarrow \mathbb{R}$ a continuous function. Let $a \in \mathbb{R}$. We say

$$
\Delta f \geq a
$$

(in the sense of support functions) if for any $p \in M$ and any $\varepsilon>0$ there is a smooth lower support function $\tilde{f}=f_{p, \varepsilon}$ (i.e. $\tilde{f} \leq f, \tilde{f}(p)=f(p)$ ) defined in a neighborhood of $p$ with

$$
\Delta \tilde{f}(p) \geq a-\varepsilon
$$

where $\Delta \tilde{f}=\operatorname{trace} D \nabla \tilde{f}=\operatorname{div} \nabla \tilde{f}$ is the Riemannian Laplace operator (Laplace- Beltrami operator. Similarly we define $\Delta f \leq a$ using smooth upper support functions. Clearly, $\Delta f \leq a$ iff $\Delta(-f) \geq-a$. A continuous function $f$ with $\Delta f \geq 0$ is called subharmonic, and if $\Delta f \leq 0, f$ is called superharmonic.

Theorem 12.6 (Hopf-Calabi Maximum Principle, [23], [4], [13])

Let $M$ be a connected Riemannian manifold and $f: M \rightarrow \mathbb{R}$ a continuous subharmonic function. Then $f$ attains no maximum unless it is constant.

Proof. If $f$ attains a maximum at $p \in M$ and is not constant on any neighborhood of $p$, we may choose a small coordinate ball $U$ around $p$ such that

$$
\partial^{\prime} U:=\{x \in \partial U ; f(x)=f(p)\}
$$

is a proper subset of $\partial U$. Now pick a smooth function with

(a) $h(p)=0$,

(b) $h<0$ on $U$,

(c) $\Delta h \geq 0$ on $U$.

In fact, $h$ can be constructed easily in the form

$$
h=e^{\alpha \phi}-1
$$


for some function $\phi$ and a sufficiently large constant $\alpha>0$ since

$$
\Delta\left(e^{\alpha \phi}-1\right)=\left(\alpha^{2}\|\nabla \phi\|^{2}+\alpha \Delta \phi\right) e^{\alpha \phi} .
$$

If $\eta>0$ is sufficiently small, we have $f+\eta h<f(p)$ on $\partial U$ while $(f+\eta h)(p)=f(p)$. This shows that $f+\eta h$ attains a maximum on $U$, say at $q$. Then also the lower support function $f_{q, \varepsilon}+\eta h$ of $f+\eta h$ at $q$ takes a maximum at $q$, but

$$
\Delta\left(f_{q, \varepsilon}+\eta h\right)(q) \geq-\varepsilon+\eta \Delta h(q)>0
$$

if we choose $\varepsilon$ sufficiently small. This is a contradiction since the Hessian of a function at a maximum point is negative semidefinite, so its trace is $\leq 0$.

Thus, the set of points where $f$ attains a maximum is open and closed and by hypothesis not empty, hence it is the whole manifold.

Proof of Theorem 12.1, equality part:

Let $p, q \in M$ with $|p, q|=\pi$. Let $\rho_{p}(x)=|x, p|, \rho_{q}(x)=|x, q|$, and $f=\rho_{p}+\rho_{q}-\pi$. By triangle inequality we have $f \geq 0$, and $f=0$ on any shortest geodesic $\gamma$ from $p$ to $q$.

Fig. 27.

Moreover, due to Ric $\geq n-1$ and the average comparison theorem 4.1, we have on $M \backslash\{p, q\}$ :

$$
\Delta \rho_{p} \leq(n-1) \cot \rho_{p}, \quad \Delta \rho_{q} \leq(n-1) \cot \rho_{q}
$$

in the sense of support functions. In fact, to prove the first inequality at some point $x \in M \backslash\{p, q\}$, we choose a shortest geodesic segment $\beta$ from $x$ to $p$ and replace $p$ by some point $p^{\prime}$ on $\beta$ close to $p$; then the distance function $\rho_{p^{\prime}}$ from $p^{\prime}$ is smooth near $x$ and satisfies the above inequality with an arbitrary small error (by Theorem 4.1), 
and by trangle inequality, $\rho_{p^{\prime}}+\left|p^{\prime}, p\right|$ is an upper support function for $\rho_{p}$. (A similar argument was used in the proof of Toponogov's Theorem 6.1, Case 2.)

Since $\rho_{q} \geq \pi-\rho_{q}$, we have

$$
\cot \rho_{q} \leq \cot \left(\pi-\rho_{p}\right)=-\cot \rho_{p}
$$

Thus

$$
\Delta f \leq(n-1)\left(\cot \rho_{p}+\cot \rho_{q}\right) \leq 0
$$

(in the sense of support functions) on $M \backslash\{p, q\}$. Since $f$ attains the maximum 0 on $\gamma$, the maximum principle applied to $-f$ gives $f \equiv 0$. Consequently, $\rho_{q}=\pi-\rho_{p}$, and any geodesic starting from $p$ meets $q$ at the distance $\pi$. Now the equality discussion of the average comparison theorem 4.1 shows $R_{V}=I$ where $V$ is the radial vector field from $p$, and hence $M$ is isometric to $S^{n}$ (where the isometry is via $\exp _{p}$ ).

Fig. 28.

The proof of Theorem 3 is quite similar. We first show the superharmonicity of the Busemann functions which corresponds to the concavity in the case of $K \geq 0$ (cf. Lemma 10.6):

Lemma 12.7 Let $M$ be complete with Ric $\geq 0$ and $\gamma:[0, \infty) \rightarrow M$ be any ray in $M$. Then the corresponding Busemann function $b_{\gamma}$ is superharmonic, i.e. $\Delta b_{\gamma} \leq 0$ in the sense of support functions.

Proof. Recall that for any $x \in M$, we have a smooth upper support function

$$
b_{x, t}(y):=\left|\gamma_{x}(t), y\right|-t+b_{\gamma}(x)
$$


of $b_{\gamma}$ at $x$, where $\gamma_{x}$ is an asymptotic ray starting at $x$ (cf. Ch.10). By the avarage comparison theorem 4.1 we have

$$
\Delta b_{x, t}(x) \leq \frac{n-1}{t}
$$

thus $\Delta b_{\gamma} \leq 0$ in the sense of support functions.

Proof of Theorem 12.2. (cf. [13])

Devide the line $\gamma$ in $M$ into the two rays $\gamma^{+}, \gamma^{-}:[0, \infty) \rightarrow M$ by putting $\gamma^{ \pm}(t)=\gamma( \pm t)$, $t \geq 0$. Let $b^{ \pm}$be the Busemann function associated to $\gamma^{ \pm}$. Then $b^{+}+b^{-} \geq 0$ by triangle inequality, with equality along $\gamma$. Moreover, by the previous lemma we have

$$
\Delta\left(b^{+}+b^{-}\right) \leq 0
$$

in the sense of support functions. Thus $b^{+}+b^{-} \equiv 0$ by the maximum principle. Moreover, for any $x \in M$,

$$
b_{x, t}^{+} \geq b^{+}=-b^{-} \geq-b_{x, t}^{-} .
$$

Thus, $b:=b^{+}$is once differentiable at $x$, and the asymptotic rays $\gamma_{x}^{+}, \gamma_{x}^{-}$fit together to a complete geodesic $\gamma_{x}$ perpendicular to the level hypersurface $\{y \in M ; b(y)=b(x)\}$. Further, let

$$
A_{t}^{ \pm}(u)=D \nabla b_{x, t}^{ \pm}\left(\gamma_{x}(u)\right)
$$

For fixed $u, A_{t}^{ \pm}(u)$ is monotonely decreasing with $t$, and bounded below since $A_{t}^{+}(u) \geq$ $-A_{t}^{-}(u)$ by (12.1). Thus $A_{t}^{ \pm}$converges to some solution $A^{ \pm}$of the Riccati equation along $\gamma_{x}$. Moreover, $A^{+} \leq-A^{-}$and trace $A^{ \pm} \leq 0$ which implies $A^{+}=-A^{-}=: A$ with $\operatorname{trace} A=0$. The Riccati equation gives

$$
\|A\|^{2}=\operatorname{trace} A^{2}=-\operatorname{Ric}\left(\gamma_{x}^{\prime}\right) \leq 0
$$

thus $A \equiv 0$. Hence $D \nabla b_{x, t}^{ \pm}(x) \rightarrow 0$ as $t \rightarrow \infty$, and therefore, $b^{ \pm}$is concave (cf. Ch.10). Since $b^{+}=-b^{-}, b$ is also convex and hence affine, i.e. $b \circ \beta=0$ for any geodesic $\beta$. Thus $b$ is smooth with $D \nabla b=0$, i.e. $\nabla b$ is a parallel vector field. Let $M^{\prime}$ denote the level hypersurface $\{b=0\}$ and $\phi_{t}$ be the flow of $\nabla b$. Then the map $\Phi: M^{\prime} \times \mathbb{R} \rightarrow M$ with

$$
\Phi(x, t)=\phi_{t}(x)=\gamma_{x}(t)
$$

is an isometry. 
There are interesting applications of Theorem 12.2 since sometimes, we get a line for free:

Corollary 12.8 Let $M$ be a complete non-compact irreducible manifold (i.e. not a Riemannian product) such that $R i c \geq 0$. Then $M$ has only one end, for any compact subset $C \subset M$, the complement $M \backslash C$ has only one unbounded connected component.

(By definition, an end of a non-compact manifold $M$ is a function $E: \mathcal{K} \rightarrow \mathcal{M}$, where $\mathcal{K}$ is the set of all compact subsets of $M$ and $\mathcal{M}$ the open ones, such that $E(C)$ is a connected component of $M \backslash C$ with $E(D) \subset E(C)$ whenever $D \supset C$.)

Proof. If we had two different unbounded connected components of $M \backslash C$, we would take diverging sequences $\left(p_{i}\right),\left(q_{i}\right)$ in each component and join $p_{i}$ to $q_{i}$ by a shortest geodesic segment $\gamma_{i}$. These geodesics have to pass through $C$, so they accumulate, and a limit geodesic is a line (since $\left|p_{i}, q_{i}\right| \rightarrow \infty$ ) which is excluded by irreducibility and Theorem 12.2.

Corollary 12.9 Let $M$ be a compact manifold with Ric $\geq 0$. Let us suppose that the universal cover $\hat{M}$ is irreducible. Then $\pi_{1}(M)$ is finite.

Proof. Assume $\hat{M}$ non-compact (otherwise we are done). Then there is a compact fundamental domain $F$ related to $\Gamma \cong \pi_{1}(M)$ (acting isometrically on $\hat{M}$ ). Fix $o \in F$. Choose a sequence $p_{i} \rightarrow \infty$ in $M$ and join $o$ to $p_{i}$ by a shortest geodesic segment $\gamma_{i}$. Let $q_{i}$ be the midpoints of $\gamma_{i}$. Since $F$ is a fundamental domain, there exist $g_{i} \in \Gamma$ such that $g_{i} q_{i} \in F$. Thus, as in the proof of Corollary 12.8, the shortest geodesic segments $g_{i} \gamma_{i}$ accumulate to a line $\gamma$. This is impossible by Theorem 12.2 and the irreducibility of $\hat{M}$. 


\section{The Bochner technique.}

Let $M$ be a Riemannian manifold and consider a vector field $V$ on M. In Ch.2, we considered the derivative $A=D V$ and derived the equation

$$
D_{V} A+A^{2}+R_{V}=D W
$$

with $W=D_{V} V$ (cf. (2.5)). Taking the trace, we get

$$
\partial_{V} \operatorname{div}(V)+\operatorname{trace}\left(A^{2}\right)+\operatorname{Ric}(V)=\operatorname{div}(W)
$$

(Recall that the divergence of a vector field $X$ is given by $\operatorname{div}(X)=\operatorname{trace} D X$.) Suppose now $M$ is compact with no boundary, then

$$
\int_{M}\left(\partial_{V} \operatorname{div}(V)+\operatorname{trace}\left(A^{2}\right)+\operatorname{Ric}(V)\right)=0 .
$$

by the Divergence Theorem (since $\partial M=\emptyset)$.

Theorem 13.1 (Bochner)

Let $M$ be a compact manifold without boundary.

a) If $A$ is symmetric, $\operatorname{div}(V)=0$ and $\operatorname{Ric}(V) \geq 0$, then

$$
D V=0 \quad \text { and } \operatorname{Ric}(V)=0 .
$$

b) If $A$ is antisymmetric (in particular, $\operatorname{div}(V)=0$ ) and $\operatorname{Ric}(V) \leq 0$, then

$$
D V=0 \quad \text { and } \operatorname{Ric}(V)=0 .
$$

Proof. This is clear by (13.2): Since the first term vanishes by hypothesis, the integrand does not change sign, so it must vanish pointwise.

Corollary 13.2 Let $M$ be compact with $\operatorname{Ric}(V)>0$. Then the first Betti number $b_{1}(M)$ vanishes.

Proof. Since the first de-Rham cohmomology of $M$ is

$$
H^{1}(M)=\{\text { local gradients }\} /\{\text { gradients }\},
$$

we only have to show that each local gradient vector field $V$ (i.e. $D V=(D V)^{*}$, cf. Ch.2) is the gradient of some function. Put $\tilde{V}=V-\nabla f$ where $f: M \rightarrow \mathbb{R}$ is a solution of the equation $\Delta f=\operatorname{div}(V)$. Then $\tilde{V}$ is still a local gradient, and moreover, $\operatorname{div}(\tilde{V})=0$. Thus Theorem 13.1 a) applies to $\tilde{V}$, and now we get the contradiction $\operatorname{Ric}(\tilde{V})=0$, unless $\tilde{V}=0$, i.e. $V=\nabla f$, so $V$ is a gradient. 
Corollary 13.3 Let $M$ be compact with Ric $<0$. Then the group of isometries of $M$ is finite.

Proof. It is known (cf. [26]) that $I(M)$, the group of isometries of $M$, is a compact Lie group. Any one-parameter subgroup $\left(g_{t}\right)_{t \in \mathbb{R}}$ of $I(M)$ gives rise to a vector field $V$ on $M$, defined by

$$
V(x)=\left.\frac{d}{d t} g_{t}(x)\right|_{t=0}
$$

which is the Killing field corresponding to $\left(g_{t}\right)$. Since $g_{t}$ is an isometry,

$$
\left\langle\left(d g_{t}\right)_{x} \cdot a,\left(d g_{t}\right)_{x} \cdot b\right\rangle=\langle a, b\rangle
$$

for all $a, b \in T_{x} M$. Differentiating with respect to $t$ we see that $\left\langle D_{a} V, b\right\rangle+\left\langle a, D_{b} V\right\rangle=0$, i.e. $A=D V$ is skew symmetric. (In fact, this property characterizes Killing fields.) Now from Theorem $13.1 \mathrm{~b})$ we get $\operatorname{Ric}(V)=0$ which is a contradiction to Ric $<0$ unless $V=0$. So there are no nontrivial one parameter subgroups in $I(M)$. Hence $I(M)$ is discrete; since it is also compact, it must be finite.

Remark 13.4 Corollaries 13.2 and 13.3 can be easily extended to the case where Ric $\geq 0$ or Ric $\leq 0$. Then applying 13.1 to a divergence-free local gradient field (Case (a)) or to a Killing field (Case (b)) $V$, we recieve $D V=0$. In Case (a) we recieve that the first Betti number is the number of linear independent parallel vector fields on $M$, so in particular, $b_{1}(M) \leq n$, and in Case (b) we get that any Killing field is parallel, so the connected component of $I(M)$ acts only on a flat factor of $M$.

Remark 13.5 It is interesting to compare the proofs of Corollaries 13.1 (Bochner) and 12.2 (Myers) which both show that a compact manifold $M$ with Ric $>0$ has $b_{1}(M)=0$. (Recall that by Hurewitz, $b_{1}(M)=0$ if $\pi_{1}(M)$ is finite, but the converse does not hold.) In both proofs we have used the equation

$$
\partial_{V} \operatorname{trace}(A)+\operatorname{trace}\left(A^{2}\right)+\operatorname{Ric}(V)=\operatorname{div}(W)
$$

for a local gradient vector field $V$, where $A=D V$ and $W=D_{V} V$. However, in Myers' theorem, we have assumed $W=0$ and estimated trace $A$ (as a solution of the Riccati equation) while in Bochner's theorem, we have assumed $\operatorname{trace} A=0$ and allowed arbitrary $W$. Myers' result is stronger for Ric $>0$, however, Bochner's technique gives a result also for $R i c \geq 0$. 


\section{Appendix: Nested Coverings}

In this appendix, we want to prove the topological result on coverings which we have used in Ch.8, cf. (8.20). The following exposition is essentially due to U. Abresch (cf. [28]). Let us first recall the Mayer-Vietoris principle (cf [3]). Let $X=X_{1} \cup \ldots \cup X_{N}$ be a topological space. Let $S X$ denote the complex of singular chains $c=\sum_{i} \alpha_{i} \sigma_{i}$ where $\sigma_{i}: \Delta^{q} \rightarrow X$ are singular $(q-)$ simplices and $\alpha_{i}$ coefficients in the chosen field $\mathbf{F}$. The homology of $X$ can be computed from the subcomplex $\tilde{S} X$ of small chains where a chain $c$ is called small if all the $\sigma_{i}$ take values in some $X_{j}$. For $N=2$ we get the usual Mayer-Vietoris exact sequence of chain complexes:

$$
\begin{aligned}
0 \rightarrow S\left(X_{1} \cap X_{2}\right) \rightarrow & S X_{1} \oplus S X_{2} \rightarrow \tilde{S} X \rightarrow 0 \\
& \left(c_{1}, c_{2}\right) \mapsto c_{1}+c_{2} \\
c \mapsto(c,-c) &
\end{aligned}
$$

For arbitrary $N$, we put

$$
X_{i_{1}, \ldots, i_{k}}:=X_{i_{1}} \cap \ldots \cap X_{i_{k}},
$$

and putting $C_{0}=\tilde{S} X, C_{1}=\sum_{i} S X_{i}, C_{2}=\sum_{i, j} X_{i j}$ etc., we get an exact sequence

$$
\rightarrow C_{3} \rightarrow C_{2} \rightarrow C_{1} \rightarrow C_{0} \rightarrow 0
$$

where the maps $\delta_{k}: C_{k} \rightarrow C_{k-1}$ are defined by their "matrix elements"

$$
\delta_{k}\left(i_{1}, \ldots, i_{k} ; j_{1}, \ldots, j_{k-1}\right): S X_{i_{1}, \ldots, i_{k}} \rightarrow S X_{j_{1}, \ldots, j_{k-1}}
$$

as follows: the only nonzero matrix elements are

$$
\delta_{k}\left(i_{1}, \ldots, i_{k} ; i_{1}, \ldots \hat{i_{j} \ldots}, i_{k}\right)=(-1)^{j-1} \cdot i n c
$$

where inc denotes the natural inclusion map. Put

$$
A_{k}=\operatorname{im} \delta_{k}=\operatorname{ker} \delta_{k-1} \subset C_{k-1} .
$$

In particular, we have

$$
A_{1}=C_{0}=\tilde{S} X
$$

The above exact sequence of chain complexes can be split into short exact sequences as follows:

$$
0 \longrightarrow A_{k+1} \longrightarrow C_{k} \stackrel{\delta_{k}}{\longrightarrow} A_{k} \longrightarrow 0 \text {. }
$$


As usual, a short exact sequence of chain complexes gives rise to a long exact sequence of the homologies:

$$
\rightarrow H_{p} A_{k+1} \rightarrow H_{p} C_{k} \rightarrow H_{p} A_{k} \rightarrow H_{p-1} A_{k+1} \rightarrow \ldots
$$

We will use only the segment

$$
H_{p} C_{k} \rightarrow H_{p} A_{k} \rightarrow H_{p-1} A_{k+1}
$$

in order to estimate the middle term. If we had only to compute the Betti numbers, we would get

$$
\operatorname{dim} H_{p} A_{k} \leq \operatorname{dim} H_{p} C_{k}+\operatorname{dim} H_{p-1} A_{k+1}
$$

for all $k$, and in particular

$$
b_{p}(X)=\operatorname{dim} H_{p} A_{1} \leq \operatorname{dim} H_{p} C_{1}+\operatorname{dim} H_{p-1} A_{2},
$$

and further

$$
\operatorname{dim} H_{p-1} A_{2} \leq \operatorname{dim} H_{p-1} C_{2}+\operatorname{dim} H_{p-2} A_{3}
$$

and so on, hence by induction

$$
b_{p}(X) \leq \operatorname{dim} H_{p} C_{1}+\operatorname{dim} H_{p-1} C_{1}+\ldots+\operatorname{dim} H_{0} C_{p+1}
$$

since $H_{p-q} A_{q+1}=0$ for $q>p$. However, these Betti numbers are not available in our application; instead, we have to compute the content which is the rank of certain inclusion maps. Unfortunately, the analogue of (A1) for the rank in place of the dimension is not true: If we have a commutative diagram of exact sequences,

$$
\begin{gathered}
A \stackrel{\phi}{\longrightarrow} B \stackrel{\psi}{\longrightarrow} C \\
\downarrow \alpha \quad \downarrow \beta \quad \downarrow \gamma \\
A^{\prime} \stackrel{\phi^{\prime}}{\longrightarrow} B^{\prime} \stackrel{\psi^{\prime}}{\longrightarrow} C^{\prime}
\end{gathered}
$$

we have not $\operatorname{rk}(\beta) \leq \operatorname{rk}(\alpha)+\operatorname{rk}(\gamma)$; e.g. we could choose $\alpha=0$ and $\gamma=0$, and $\beta$ maps $\operatorname{im}(\phi)$ onto 0 while a complement of $\operatorname{im} \phi$ is mapped into $\operatorname{ker}\left(\psi^{\prime}\right)$. However, if we have three such sequences,

$$
\begin{gathered}
A \stackrel{\phi}{\longrightarrow} B \stackrel{\psi}{\longrightarrow} C \\
\downarrow \alpha \quad \downarrow \beta \quad \downarrow \gamma \\
A^{\prime} \stackrel{\phi^{\prime}}{\longrightarrow} B^{\prime} \stackrel{\psi^{\prime}}{\longrightarrow} C^{\prime} \\
\downarrow \alpha^{\prime} \quad \downarrow \beta^{\prime} \quad \downarrow \gamma^{\prime} \\
A^{\prime \prime} \stackrel{\phi^{\prime \prime}}{\longrightarrow} B^{\prime \prime} \stackrel{\psi^{\prime \prime}}{\longrightarrow} C^{\prime \prime}
\end{gathered}
$$


then we get

$$
\operatorname{rk}\left(\beta^{\prime} \circ \beta\right) \leq \operatorname{rk}\left(\alpha^{\prime}\right)+\operatorname{rk}(\gamma)
$$

Proof. Consider a decomposition

$$
\operatorname{im}(\beta)=(\operatorname{im} \beta)_{1} \oplus(\operatorname{im} \beta)_{2}
$$

where

$$
(\operatorname{im} \beta)_{1}=\operatorname{im}(\beta) \cap \operatorname{ker}\left(\psi^{\prime}\right) .
$$

Then $(\operatorname{im} \beta)_{1} \subset \operatorname{im}\left(\phi^{\prime}\right)$, hence

$$
\beta^{\prime}(\operatorname{im} \beta)_{1} \subset \phi^{\prime \prime}\left(\alpha^{\prime}\left(A^{\prime}\right)\right)
$$

Moreover, $\psi^{\prime}$ is injective on $(\operatorname{im} \beta)_{2}$, and

$$
\psi^{\prime}(\operatorname{im} \beta)_{2} \subset \gamma(\psi(B)) .
$$

Therefore,

$$
\begin{aligned}
\operatorname{rk}\left(\beta^{\prime} \circ \beta\right) & \leq \operatorname{dim}\left(\beta^{\prime}(\operatorname{im} \beta)_{1}\right)+\operatorname{dim}\left(\beta^{\prime}(\operatorname{im} \beta)_{2}\right) \\
& \leq \operatorname{rk}\left(\alpha^{\prime}\right)+\operatorname{rk}(\gamma)
\end{aligned}
$$

We apply this to

$$
\begin{aligned}
& H_{p} C_{k}^{0} \longrightarrow H_{p} A_{k}^{0} \longrightarrow H_{p-1} A_{k+1}^{0} \\
& \downarrow \quad \downarrow \quad \downarrow \\
& H_{p} C_{k}^{p} \longrightarrow H_{p} A_{k}^{p} \longrightarrow H_{p-1} A_{k+1}^{p} \\
& \downarrow \quad \downarrow \quad \downarrow \\
& H_{p} C_{k}^{p+1} \longrightarrow H_{p} A_{k}^{p+1} \longrightarrow H_{p-1} A_{k+1}^{p+1}
\end{aligned}
$$

where $C_{k}^{p}$ and $A_{k}^{p}$ are the above defined complexes, but now for $\mathrm{p}+2$ different coverings $\left\{X_{1}^{q}, \ldots, X_{N}^{q}\right\}$ of $X(q=1, \ldots, p+2)$ with the property that

$$
X_{i}^{q-1} \subset X_{i}^{q}
$$

for $q=1, \ldots, p+1$ (nested coverings). Thus we get

$$
\begin{aligned}
& \operatorname{rk}\left(H_{p} A_{k}^{0} \rightarrow H_{p} A_{k}^{p+1}\right) \\
& \quad \leq \operatorname{rk}\left(H_{p} C_{k}^{p} \rightarrow H_{p} C_{k}^{p+1}\right)+\operatorname{rk}\left(H_{p-1} A_{k+1}^{0} \rightarrow H_{p-1} A_{k+1}^{p}\right)
\end{aligned}
$$


for all $k$ and in particular

$$
\begin{aligned}
& \operatorname{rk}\left(H_{p} A_{1}^{0} \rightarrow H_{p} A_{1}^{p+1}\right) \\
& \quad \leq \operatorname{rk}\left(H_{p} C_{1}^{p} \rightarrow H_{p} C_{1}^{p+1}\right)+\operatorname{rk}\left(H_{p-1} A_{2}^{0} \rightarrow H_{p-1} A_{2}^{p}\right)
\end{aligned}
$$

and further

$$
\begin{aligned}
& \operatorname{rk}\left(H_{p-1} A_{2}^{0} \rightarrow H_{p-1} A_{2}^{p+1}\right) \\
& \quad \leq \operatorname{rk}\left(H_{p-1} C_{2}^{p-1} \rightarrow H_{p-1} C_{2}^{p}\right)+\operatorname{rk}\left(H_{p-2} A_{3}^{0} \rightarrow H_{p-2} A_{3}^{p-1}\right)
\end{aligned}
$$

and so on. Thus we get by induction

$$
\begin{aligned}
& \operatorname{rk}\left(H_{p} A_{1}^{0} \rightarrow H_{p} A_{1}^{p+1}\right) \\
& \quad \leq \sum_{j=0}^{p} \operatorname{rk}\left(H_{p-j} C_{j+1}^{p-j} \rightarrow H_{p-j} C_{j+1}^{p+1-j}\right)
\end{aligned}
$$

Recall that

$$
\begin{aligned}
H_{q} C_{K}^{q} & \rightarrow H_{q} C_{k}^{q+1} \\
\quad= & \bigoplus_{i_{1}<\ldots<i_{k}}\left(H_{q}\left(X_{i_{1}}^{q} \cap \ldots \cap X_{i_{k}}^{q}\right) \rightarrow H_{q}\left(X_{i_{1}}^{q+1} \cap \ldots \cap X_{i_{k}}^{q+1}\right) .\right.
\end{aligned}
$$

We apply this to our coverings of balls $B_{i}^{j}=10^{j} B_{i}$ for $j=0, \ldots, n+1$ and recieve

$$
\begin{aligned}
\operatorname{cont} & \left(\bigcup_{i} B_{i}, \bigcup_{i} 10^{n+1} B_{i}\right) \\
& =\sum_{p=0}^{n} \operatorname{rk}\left(H_{p}\left(\bigcup_{i} B_{i}^{0}\right) \rightarrow H_{p}\left(\bigcup_{i} B_{i}^{n+1}\right)\right) \\
& \leq \sum_{p=0}^{n} \operatorname{rk}\left(H_{p}\left(\bigcup_{i} B_{i}^{n-p}\right) \rightarrow H_{p}\left(\bigcup_{i} B_{i}^{n+1}\right)\right)
\end{aligned}
$$

We estimate each term individually by (A2): For given $p$ we put

$$
X=\bigcup_{i} B_{i}^{n-p}, \quad X_{i}^{q}=B_{i}^{n-p+q}
$$

and sum over $p$. Putting now

$$
C_{k}^{q}=\sum_{i_{1}<\ldots<i_{k}} S\left(B_{i_{1}}^{q} \cap \ldots \cap B_{i_{k}}^{q}\right)
$$


and

$$
\gamma_{p, k}^{q}: H_{p} C_{k}^{q} \rightarrow H_{p} C_{k}^{q+1}
$$

we get from $(\mathrm{A} 2)$

$$
\begin{aligned}
& \operatorname{cont}\left(\bigcup_{i} B_{i}, \bigcup_{i} 10^{n+1} B_{i}\right) \\
& \leq \quad \quad \quad r k \gamma_{n, 1}^{n}+\operatorname{rk} \gamma_{n-1,2}^{n-1}+\ldots+\operatorname{rk} \gamma_{1, n}^{1}+\operatorname{rk} \gamma_{0, n+1}^{0} \\
& +\operatorname{rk} \gamma_{n-1,1}^{n}+\operatorname{rk} \gamma_{n-2,2}^{n-1}+\ldots+\operatorname{rk} \gamma_{0, n}^{1} \\
& +\quad \cdots \\
& +\quad \mathrm{rk} \gamma_{1,1}^{n}+\operatorname{rk} \gamma_{0,2}^{n-1} \\
& +\quad \mathrm{rk} \gamma_{0,1}^{n} \\
& \leq \quad \quad \quad \quad r k \gamma_{*, 1}^{n}+\operatorname{rk} \gamma_{*, 2}^{n-1}+\ldots+\operatorname{rk} \gamma_{*, n}^{1}+\operatorname{rk} \gamma_{*, n+1}^{0}
\end{aligned}
$$

Hence

$$
\begin{aligned}
\operatorname{cont} & \left(\bigcup_{i} B_{i}, \bigcup_{i} 10^{n+1} B_{i}\right) \\
\leq & \sum_{k=1}^{n+1} \mathrm{rk} \gamma_{*, k}^{n+1-k} \\
& =\sum_{k=1}^{n+1} \sum_{i_{1}<\ldots<i_{k}} \operatorname{cont}\left(\bigcap_{j=1}^{k} B_{i_{j}}^{n+1-k}, \bigcap_{j=1}^{k} B_{i_{j}}^{n+2-k}\right)
\end{aligned}
$$

which proves Equation (8.20).

\section{References:}

[1] Abresch, U. Lower curvature bounds, Toponogov's theorem and bounded topology I,II. Ann. scient. Éc. Norm. Sup., $4^{e}$ série, t.18 (1985), 651-670

[2] Berger, M.: Les variétés riemanniennes (1/4)-pincées. Ann. Sc. Norm. Super. Pisa, III 14 (1960), 161-170

[3] Bott, R., Tu, L.W.: Differential forms in algebraic topology. Springer Graduate Texts in Mathematics 82 (1982)

[4] Calabi, E.: An extension of E.Hopf's maximum principle with an application to Riemannian geometry. Duke Math. J. 25 (1957), 45-56 
[5] Cheeger, J., Ebin, D.G.: Comparison theorems in Riemannian geometry American Elsevier, New York 1975

[6] Cheeger, J., Gromoll, D.: On the structure of complete manifolds of nonnegative curvature. Ann. of Math. 96 (1972), 413-442

[7] Cheeger, J., Gromoll, D.: The splitting theorem for manifolds of nonnegative Ricci curvature. J. Diff. Geom. 6 (1971), 119-128

[8] Cheng, S.Y.: Eigenvalue comparison theorem and its geometric application. Math. Z. 143 (1975), 289-297

[9] Eschenburg, J.-H. Comparison theorems and hypersurfaces. Manuscripta math., 59 (1987), 295-323

[10] Eschenburg, J.-H. Diameter, volume, and topology for positive Ricci curvature. Journal of Differential Geometry, 33 (1991), 743-747

[11] Eschenburg, J.-H. Local convexity and nonnegative curvature - Gromov's proof of the sphere theorem. Invent. math., 84 (1986), 507-522

[12] Eschenburg, J.-H. Maximum Principle for Hypersurfaces, Manuscripta Math. 64 (1989), $55-75$

[13] Eschenburg, J.-H., Heintze, E. An elementary proof of the Cheeger - Gromoll splitting theorem. Ann. Glob. Analysis and Geometry, vol. 2, n. 2 (1984), 141-151

[14] Eschenburg, J.-H., Heintze, E. Comparison theory for Riccati equation Manuscripta math., 68 (1990), 209-214

[15] Green, L.W.: A theorem of E.Hopf. Mich. Math. J. 5 (1958), 31-34

[16] Greene, R.E., Wu, H.: On the subharmonicity and plurisubharmonicity of geodesically convex functions. Ind. Univ. Math. J. 22 (1973), 641-653

[17] Greene, R.E., Wu, H.: $C^{\infty}$-convex functions and manifolds of positive curvature. Acta Math. 137 (1976), 209-245

[18] Gromoll, D., Klingenberg, W., Meyer, W.: Riemannsche Geometrie im Großen Lecture notes 55, Springer 1968 
[19] Gromov, M.: Curvature, diameter and Betti numbers. Comm. Math. Helv. 56 (1981), 179-195

[20] Gromov, M.: Structures métriques pour les variétés riemanniennes. Cedic-Nathan 1981

[21] Grove, K., Shiohama, A.: A generalized sphere theorem. Ann. of Math. 106 (1977), 201-211

[22] Hadamard, J.: Sur certain propriétés des trajectories en dynamique. J. Math. Pures Appl. (5)3 (1897), 331-387

[23] Hopf, E.: Elementare Bemerkungen über die Lösungen partieller Differentialgleichungen 2.Ordnung vom elliptischen Typ. Sitzungsber. Preuss. Ak. d. Wiss. 19 (1927), 147-152

[24] Karcher, H.: Riemannian comparison constructions. In: S.S.Chern (ed.): Studies in Global Geometry and Analysis, M.A.A. Studies in Mathematics, vol. 27 (1989)

[25] Klingenberg, W.: Über Riemannsche Mannigfaltigkeiten mit positiver Krümmung. Comm. Math. Helv. 35 (1961), 47-54

[26] Kobayashi, S., Nomizu, K.: Foundations of differential geometry, I. Interscience, Wiley, New York 1963

[27] Kürzel, M. Der Vergleichssatz von Toponogov im Fall variabler Krümmung. Diplomarbeit, Münster 1994

[28] Meyer, W. Toponogov's theorem and applications. Preprint University of Münster (1990)

[29] Milnor, J.: Morse Theory. Princeton 1962

[30] Milnor, J.: A note on curvature and fundamental group. J. Diff. Geom. 2 (1968), $1-7$

[31] Myers, S.B.: Riemannian manifolds with positive mean curvature. Duke Math. J. 8 (1941), 401-404

[32] Sharafutdinov, V.A.: Convex sets in a manifold of nonnegative curvature. Math. 
Zametki 26 (1979) 556-560

[33] Strake, M., Walshap, G. : $\Sigma$-flat manifolds and Riemannian submersions. Manuscr. Math. 64 (1989), 213-226

[34] Wu, H.: An elementary method in the study of nonnegative curvature. Acta Math. 142 (1979), 57-78

[35] J.-W.Yim: Distance nonincreasing retraction on a complete open manifold of nonnegative sectional curvature. Ann. Glob. Anal. and Geom. 


\section{Contents}

0. Introduction. 1

1. Covariant derivative and curvature. 2

2. Jacobi and Riccati equations; equidistant hypersurfaces. 6

3. Comparison theory. 12

4. Average comparison theoremx. 15

5. Bishop-Gromov inequality 17

6. Toponogov's triangle comparison theorem 21

7. Number of generators and growth of the fundamental group 26

8. Gromov's estimate of the Betti numbers 31

9. Convexity 39

10. Open manifolds with nonnegative curvature 43

11. The sphere theorem 50

12. Lower Ricci curvature bounds and the Maximum Principle. 59

13. The Bochner technique. 65

Appendix: Nested coverings 67

References 72

Contents 75

Index 76 


\section{Index}

affine function 63

Betti numbers 30

Bishop-Gromov inequality 17,20

Busemann function 43,44,62

Bochner 65

comparison theorem 12

complete 5

compressible 33

concave 39,44

conjugate points 18

content 32

convex $39,43,52$

convex hypersurface 40

corank 34

critical points $31,33,34$

curvature tensor 3

cut locus 17

diameter 26

distance 2,9

divergence 63

end 64

equidistant hypersurfaces 9,13

exponential coordinates 5

exponential map 5,18

focal points 9

free action 26

fundamental group 26,59,64

fundamental domain 26

Gauss map 52

geodesic 4

gradient 2

gradientlike 30

growth of a group 28,29

Hopf-Calabi maximum principle 60

Hopf-Rinow theorem 17,19

Hadamard 52

horospheres 43

incompressible 34 
in the sense of support functions 39,60

Jacobi field 6

Killing field 66

$\mathrm{k}(\mathrm{p}, \mathrm{r}) 34$

Laplacian 60

length 2

Levi-Civita derivative 2

line 59

local gradient 8,65

Mayer-Vietoris principle 67

mean curvature 9,15

Morse theory 30,34

normal bundle 43

open manifold 43

parallel 4,12,63

Perelman's theorem 47

principal curvatures 9,13

ray 43

regular point 31

Riccati equation 8,12

Riemannian manifold 2

Riemannian product 59

Ricci curvature 3

rigidity theorem 60

scalar curvature 3

sectional curvature 3

shape operator 9

Sharafutdinov 47,49

shortest curve 2,17

soul 43

sphere theorem 50

standard space 19

subharmonic 60

superharmonic 60

support function 25,39

Toponogov theorem 22

totally geodesic 14

umbilical 10

vector field along a map 4 